\title{
Modeling of polyolefin polymerization in semibatch slurry reactors: experiments and simulations
}

Tommaso Casalini ${ }^{1}$, Frans Visscher ${ }^{2}$, Erik Janssen ${ }^{2}$, Francesco Bertola ${ }^{2}$, Giuseppe Storti ${ }^{1}$ and Massimo Morbidelli ${ }^{1, *}$

${ }^{1}$ Institute for Chemical and Bioengineering, Department of Chemistry and Applied Biosciences, ETH Zurich, Zurich, Switzerland

${ }^{2}$ SABIC, Technology, Geleen, The Netherlands

*corresponding author; email: massimo.morbidelli@chem.ethz.ch

\begin{abstract}
In this work, a well-known single-particle model (the multigrain model) is applied to simulate size growth and morphology evolution of polyethylene particles obtained through a slurry-phase, catalytic polymerization in semibatch reactor. The model explicitly takes into account diffusion limitations within the polymer particle and between the particle and the solvent, as well as void fraction variations due to the particle growth. Catalyst behavior is described assuming a two-site model, which represents a good compromise between accuracy and simplicity, along with a conventional kinetic scheme, whose kinetic parameters are determined by fitting the model predictions to the experimental data. Polymer molecular weight is evaluated from population balances, solved through the method of moments in order to reduce the computational effort. The semibatch reactor is modeled by means of fundamental mass conservation equations. Simulations showed that the proposed model is able to properly describe not only the polymer properties of interest (such as average molecular weight and polydispersity) but also the evolution of the average particle size, thus providing a comprehensive overview of the system behavior. The model is further validated through regression-free simulation of additional sets of experimental data, including the case of materials with a bimodal molar mass distribution: the good agreement found also in this case confirms the robustness of the model and the estimated parameter values.
\end{abstract}

\section{Introduction}

Although the catalytic polymerization of polyolefins in gas or slurry phase is nowadays an established reality $^{[1]}$, the development of mathematical models of the overall process at the single catalyst particle scale is still underway ${ }^{[2]}$. Even though the first modeling attempt dates back to 1971 with the work of Singh and Merrill ${ }^{[3]}$, more comprehensive models are continuously proposed in order to improve the description of the involved phenomena ${ }^{[4]}$. The final goal of this body of work is the development of comprehensive singleparticle models which allow predicting the final properties of interest of the polymer (molecular weights, polydispersity, rheological properties, copolymer composition when comonomers are involved, et cetera) as well as particle morphology (size, porosity, et cetera) as a function of operating conditions. Coupled with an 
appropriate mathematical description of the reactor, such models represent a valuable tool for process optimization, i.e. improving plant efficiency and product quality.

At single particle level, several models have been proposed in the literature ${ }^{[5-7]}$, such as the polymeric flow model ${ }^{[3,8]}$, the multigrain model ${ }^{[9,10]}$ or the random-pore polymeric flow model ${ }^{[4]}$. All these models aim to describe the complex interplay between different phenomena such as initial catalyst fragmentation, diffusion of the reactants from the liquid phase to the polymer particle and to the catalytic site, sorption equilibria for the different species among the different phases, catalyst deactivation and particle morphology evolution. Broadly speaking, such modeling efforts propose a diffusion/reaction approach where the average molecular weight of the formed polymer is usually described through the method of moments ${ }^{[11]}$ solving the corresponding population balances for growing and dead polymer chains.

The modeling of the process becomes more complex when also the reactor is taken into account in the overall description. Indeed, the mixing regime inside the reactor can lead to particles breakage or aggregation $^{[12]}$, or transport limitations at the solid/gas interface (e.g. when fluidized beds are used) can cause particle overheating because of inefficient heat removal. Moreover, when continuous reactors (e.g. single reactors or series) are employed, the residence time distribution affects the final particle size distribution ${ }^{[13]}$ and can create inhomogeneity in the polymer properties ${ }^{[14]}$. The addition of comonomers or inerts can affect the monomer solubility in the polymer, thus affecting the overall propagation rate $\mathrm{e}^{[15,16]}$.

The importance of the modeling of catalytic polyolefin polymerization processes is proved by the wide number of examples provided by the literature, where many different modeling approaches have been proposed, starting from single particle models ${ }^{[4,10]}$ that are subsequently coupled with the description of slurry phase ${ }^{[17-20]}$ or gas phase ${ }^{[21,22]}$ reactors. In continuous reactors, the impact of residence time distribution on particle size distribution can be accounted for ${ }^{[23,24]}$; in addition, population balances and appropriate kernels ${ }^{[12 \text {, }}$ ${ }^{22]}$ are used to take into account particle breakage and aggregation. Recently, computational fluid dynamics has been applied for a detailed description of polymerization processes in fluidized bed reactors, accounting for polymerization kinetics, particle breakage/aggregation and velocity field ${ }^{[25,26]}$.

The choice of the number of active sites for modeling the catalyst is a debated aspect. From a practical point of view, the minimum number of active sites is obtained through the deconvolution of molecular weight distribution ${ }^{[27]}$ into weighted summation of individual Flory distribution components. Literature offers a wide range of examples, where two ${ }^{[17,22,23]}$, three $e^{[20]}$, four ${ }^{[19,28,29]}$, five $e^{[30,31]}$ and $\operatorname{six}^{[32]}$ different active sites have been employed. A multisite modeling approach leads to an accurate description of the molecular weight distribution, but it requires the estimation of a correspondingly large set of kinetic parameters, since each active site has, in principle, its own set of kinetic constants. The fitting procedure can be non-trivial ${ }^{[33]}$ due e.g. to the presence of several local minima in the objective function to be minimized.

In this work, a model suitable to simulate the semibatch polymerization of ethylene in liquid slurry is developed and validated by comparison with a comprehensive set of experimental data. The multigrain model has been chosen as reference single-particle modeling approach, since it provides a detailed description of the many involved phenomena while enabling the prediction of the particle size and morphology evolution; 
moreover, catalyst has been described through a two-site model, in order to keep a balance between computational simplicity and a satisfactorily description.

In particular, in this work we show that a simple two-site model for catalyst, along with kinetic parameters fitted against propagation rate and molecular weights as a function of polymerization time, is enough to properly describe system behavior in terms of polymer average properties of interest over a different range of operative conditions.

In the first part of the paper, the main kinetic constants are fitted against experimental data, in order to properly reproduce polymer molecular weight as well as particle size. Then, the reliability of the model is assessed through genuinely predictive simulations of independent experimental data, in order to conclusively prove the robustness of the estimated parameters and the overall applicability of the model itself.

\section{Experimental methods}

Chemicals

Hexane isomers (supplied by Exxon-Mobil) are used as diluent after suitable purification (initial boiling point $69^{\circ} \mathrm{C}$ ). Ethylene and hydrogen are purified upon delivery. The Ziegler-Natta catalyst is prepared in-house.

\section{Reactor and flow meters}

The installation used is a $20 \mathrm{dm}^{3}$, non-baffled, stainless steel reactor supplied by Büchi (Switzerland), with inner diameter of $284 \mathrm{~mm}$ and maximum height of $585 \mathrm{~mm}$. The reactor is equipped with a 4-bladed pitch blade turbine stirrer (diameter of $120 \mathrm{~mm}$ ), mounted such that down pumping behavior is achieved. The clearance between the stirrer and the reactor bottom is $70 \mathrm{~mm}$.

A typical experiment is carried out at a rotational speed of 750 RPM, for which the presence of gasliquid mass transfer limitations has been ruled out under the given reaction conditions. Hydrogen is dosed via a Bronkhorst thermal mass flow meter with an operating range of $0-4 \mathrm{ndm}^{3} / \mathrm{hr}$ or $0-160 \mathrm{ndm} / \mathrm{hr}$. Ethylene is fed with a Bronkhorst with a range of 0-3000 ndm³/hr. Nitrogen is dosed via a pressure reducer.

\section{Control strategy}

The reactor setup includes a pressure-controlled ethylene feed to achieve the set point of the total pressure. The feed profile of ethylene $\left(\mathrm{g} \mathrm{h}^{-1}\right)$ is measured as a function of time and is representative of the polymerization rate profile as fresh ethylene is added to make up the ethylene converted to polymer in the reactor while maintaining constant pressure. The headspace composition is continuously sampled via an online GC. The targeted hydrogen-to-ethylene ratio is obtained by adjusting the feed rate of hydrogen.

The reactor temperature is kept constant at the desired value with good accuracy (within $\pm 0.5^{\circ} \mathrm{C}$ ) with the exception of a few minutes at the beginning of the reaction, when it exhibits a 1 to $2^{\circ} \mathrm{C}$ increase due to the high initial rate of polymerization. 


\section{Reaction experiment}

Starting from an empty reactor under nitrogen, $10 \mathrm{dm}^{3}$ of diluent are added. Cocatalyst is dosed such that the desired concentration is reached, after which the reactor is heated to $82.5^{\circ} \mathrm{C}$. In the next step, the reactor is vented to the vapor pressure of the diluent, followed by flushing with hydrogen. Next, the reactor is preconditioned by feeding ethylene and hydrogen. The catalyst is dosed as a suspension in diluent with nitrogen overpressure via a catalyst dosing vessel, mounted on the lid of the reactor, after which the dosing line is flushed with $10 \mathrm{~cm}^{3}$ of diluent. When the monomer consumption by reaction begins, the ethylene dosage to the reactor is started.

After polymerizing for three hours the reactor is flashed off under nitrogen to the vapor pressure of the diluent which reduces ethylene and hydrogen present in the reactor. Cooling the reaction mixture to $32^{\circ} \mathrm{C}$ terminates the first step of the polymerization.

After heating up to $79^{\circ} \mathrm{C}$, monomer and hydrogen are fed to start polymerization of the second reaction step. Based on the cumulative amount of ethylene fed during step 1, the targeted ratio between polymer produced in step 1 and step 2 can be adjusted by the duration of polymerization during in the second step. When reaching the desired polymerization time of the second step, nitrogen is used to transfer the reaction mixture into a filter where solid-liquid separation is performed.

\section{Characterization techniques}

The molecular weight was obtained from SEC-DV measurements. A Polymer Laboratories PLGPC220 was used in combination with three detectors: a Polymer Laboratories PL BV-400 viscometer, a refractive index detector; and a Polymer Char IR5 infrared detector. Three columns from Polymer Laboratories $13 \mu \mathrm{m}$ PLgel Olexis, 300x7.5 mm, were used.

\section{Model development}

Model equations are summarized below under the main assumption of isothermal system. In fact, previous literature results ${ }^{[9]}$ showed that temperature gradients are negligible in slurry polymerization, while concentration gradients can play a key role in the overall behavior of the system. Therefore, material balances only will be considered in the following.

\section{Kinetic scheme for ethylene polymerization}

The proposed kinetic scheme is well assessed in literature ${ }^{[10,17-19,29]}$ and involves catalyst activation through cocatalyst, initiation, propagation, chain transfer to monomer, chain transfer to hydrogen, and spontaneous catalyst deactivation; for the sake of simplicity, and in order to minimize the number of parameters to be estimated, spontaneous chain transfer and chain transfer to catalyst have not been considered as possible kinetic pathways. According to experimental observations, hydrogen causes a decrease in catalyst activity. The inhibition of catalyst activity has been reported by Soares and McKenna ${ }^{[34]}$, who showed that the addition of hydrogen decreases ethylene polymerization rate in semibatch reactor process. This effect also seems to be 
reversible, since the polymerization rate can be restored to its original value (i.e., before hydrogen addition) if reactor gas contents are vented off and the vessel is subsequently pressurized with pure ethylene. The activity inhibition by hydrogen during ethylene polymerization with Ziegler-Natta catalyst has been observed also by Alizadeh et al. ${ }^{[16]}$, Gemoets et al. ${ }^{[35]}$, Garoff et al. ${ }^{[36]}$ and by Kissin and coworkers ${ }^{[37-39]}$. Broadly speaking ${ }^{[34]}$, there are two mechanisms which can explain hydrogen effect on ethylene polymerization.

The simplest one assumes that the metal hydride site resulting from chain transfer reaction to hydrogen is less active toward ethylene insertion. As a consequence, increasing hydrogen concentration enhances the amount of less reactive active sites, which can be reactivated by monomer.

The alternative mechanism (proposed by Kissin ${ }^{[37-39]}$ ) assumes that active sites which have one single ethylene unit form a stable complex between the metal center and the hydrogen attached to the $\beta$-carbon atom in the chain through $\beta$-agostic interactions. The net effect is the presence of dormant sites which can be formed after ethylene insertion in a metal hydride site; again, increasing hydrogen concentration implies a higher concentration of dormant sites.

Our kinetic experimental data are not adequate to make a fully convincing and robust choice between the proposed mechanisms. Each mechanism also implies the introduction in the kinetic scheme of new reaction pathways (such as $\beta$-hydride elimination, initiation of less reactive sites, $\beta$-agostic interactions, and so on) with the estimation of the relative kinetic constants for each site.

This effect is included in the overall kinetic scheme through a reversible hydrogen adsorption on catalyst sites (as proposed by Gemoets et al. ${ }^{[35]}$ ), which is assumed to be instantaneous because of fast diffusion of hydrogen into the catalyst particles. The outcome of this inhibition is the decrease of the fraction of potential active sites initially available for the polymerization reactions.

Moreover, a two-site model is adopted; accordingly, the catalyst is modeled using two different kinds of active sites, each one with its own set of kinetic constants. Molecular weight distribution deconvolution ${ }^{\text {[27] }}$ into weighted summation of individual Flory distribution components is usually employed in order to determine the minimum number of active sites needed to describe the broadness of the experimental distributions. From a modeling point of view, up to 6 different sites have been considered [17, 19, 23, 28, 30-32] Although this approach provides an accurate description of the molecular weight distribution, several parameters must be estimated, since in principle each active site has its own set of kinetic constant. The estimation of such a large set of parameters is challenging because of the presence of several local minima of the objective function.

In this work, the primary focus is on the overall polymerization rate and on the average polymer properties (molecular weights and polydispersity). On one side, a two-site model will be proved to provide a satisfactorily description of the system in terms of average properties, while on the other site such approach minimizes the number of kinetic parameters to be evaluated and thus improves the reliability of the model predictions.

The kinetic scheme is summarized in Table 1 , using the following notation: $C_{p}{ }^{k}$ is the amount of potential active sites of type k not yet activated, [cocat] the cocatalyst concentration on crystal surface, $P_{0}{ }^{k}$ a 
vacant active site of type $\mathrm{k},[M]$ the monomer concentration on catalyst crystal surface, $P_{n}{ }^{k}$ a growing chain of length $n$ on a site of type $\mathrm{k}, D_{n}{ }^{k}$ a dead chain of length $n$ produced on a site of type $\mathrm{k},\left[\mathrm{H}_{2}\right]$ the hydrogen concentration at the catalyst crystal surface, $P_{\text {tot }}^{k}$ the sum of vacant and occupied active sites of type $k, C_{d}{ }^{k}$ the amount of deactivated sites of type $k$ and $C_{p H 2}$ the amount of active sites inhibited by hydrogen. All these quantities are expressed as mol mol ${ }_{\mathrm{Me}}{ }^{-1}$, except for $[M]$ and $\left[\mathrm{H}_{2}\right]$ whose units are $\mathrm{mol} \mathrm{cm}_{\mu \mathrm{p}}{ }^{-3}$; $\mu$ p subscript is referred to polymer microparticle, as explained in the next section (vide infra).

\begin{tabular}{|c|c|c|c|}
\hline \multicolumn{2}{|c|}{ Reaction } & Reaction rate & Kinetic constant \\
\hline Activation & $C_{p}^{k}+[$ cocat $] \rightarrow P_{0}^{k}$ & $r_{a c t}^{k}=k_{a c t}^{k} C_{p}[$ cocat $]$ & $k_{a c t}^{k}$ \\
\hline Initiation & $P_{0}^{k}+[M] \rightarrow P_{1}^{k}$ & $r_{i n}^{k}=k_{i n}^{k} P_{0}^{k}[M]$ & $k_{i n}^{k}$ \\
\hline Propagation & $P_{n}^{k}+[M] \rightarrow P_{n+1}^{k}$ & $r_{p}^{k}=k_{p}^{k} P_{n}^{k}[M]$ & $k_{p}^{k}$ \\
\hline $\begin{array}{c}\text { Chain transfer to } \\
\text { monomer }\end{array}$ & $P_{n}^{k}+[M] \rightarrow P_{1}^{k}+D_{n}^{k}$ & $r_{t M}^{k}=k_{t M}^{k} P_{n}^{k}[M]$ & $k_{t M}^{k}$ \\
\hline $\begin{array}{c}\text { Chain transfer to } \\
\text { hydrogen }\end{array}$ & $P_{n}^{k}+\left[H_{2}\right] \rightarrow P_{0}^{k}+D_{n}^{k}$ & $r_{t H}^{k}=k_{t H}^{k} P_{n}^{k}\left[H_{2}\right]$ & $k_{t H}^{k}$ \\
\hline $\begin{array}{c}\text { Spontaneous catalyst } \\
\text { deactivation }\end{array}$ & $P_{t o t}^{k} \rightarrow C_{d}^{k}$ & $r_{\text {deact }}^{k}=k_{\text {deact }}^{k} P_{*}^{k}$ & $k_{\text {deact }}^{k}$ \\
\hline $\begin{array}{l}\text { Instantaneous catalyst } \\
\text { inhibition by hydrogen }\end{array}$ & $C_{p}^{k}+\left[H_{2}\right] \leftrightarrow C_{p H_{2}}^{k}$ & - & - \\
\hline
\end{tabular}

Table 1. Summary of the applied kinetic scheme.

\section{Modeling of particle growth and morphology}

The particle growth has been described through the multigrain model, widely discussed and assessed in the literature ${ }^{[1,10,40,41]}$. In particular, our starting point is the model formulation proposed by Hutchinson et $a .^{[10]}$. Because of the limited broadening of the catalyst size distribution, inlet particles are considered as monodisperse. In other words, it is assumed that all the particles have the same initial size, which is equal to the mean value determined experimentally. Since particle growth is the same for identical particles (monomer concentration is assumed to be equal in every point of the reactor), the final polymer particles will be also identical, and thus monodisperse in size.

Multigrain model adopts a multiscale approach, since it considers a mass balance for the polymer microparticle (that is, the catalyst fragment surrounded by the growing polymer shell) and a mass balance for the polymer macroparticle, composed by many microparticles held together by the produced polymer. Assuming that the microparticles have spherical shape, the mass balance for a generic monomer can be written as follows:

$\frac{\partial\left[M_{i}\right]_{\mu p}}{\partial t}=\frac{1}{r_{\mu p}^{2}} \frac{\partial}{\partial r_{\mu p}}\left(D_{i, \mu p} r_{\mu p}^{2} \frac{\partial[M]_{\mu p}}{\partial r_{\mu p}}\right)$ 
where $\left[M_{i}\right]_{\mu p}$ is the concentration of the $i$-th monomer species in the microparticle, $r_{\mu p}$ the microparticle radial coordinate and $D_{i, \mu p}$ the diffusion coefficient of the $i$-th monomer species in the microparticle. These material balances are coupled with the corresponding boundary conditions, considering the monomer consumption by reaction at the microparticle center (eq. 2), where the catalyst crystal is located, and the attainment of equilibrium between the monomer in the polymer phase and that in the macroparticle liquid-filled pores at the microparticle surface (eq. 3).

$\begin{array}{ll}r_{\mu p}=r_{c r y s} & \left.4 \pi r_{c r y s}^{2} D_{i, \mu p} \frac{\partial\left[M_{i}\right]_{\mu p}}{\partial r_{\mu p}}\right|_{r_{\mu p}=r_{c r y s}}=\frac{4}{3} \pi r_{c r y s}^{3} R_{p, i} \\ r_{\mu p}=R_{\mu p} & {\left[M_{i}\right]_{\mu p}=\eta_{i}\left[M_{i}\right]_{m p}}\end{array}$

where $r_{\text {crys }}$ is the radius of catalyst crystal (also assumed to be of spherical shape), $R_{p, i}$ the consumption rate of the $i$-th species, $R_{\mu p}$ the microparticle radius, $\eta_{i}$ the partition factor for the $i$-th species and $\left[M_{i}\right]_{m p}$ the concentration of the $i$-th species in the macroparticle liquid-filled pores.

By applying the Quasi Steady State Assumption (QSSA) and assuming that the diffusion coefficient is constant ${ }^{[10]}$, eq. 1 can be analytically solved with the boundary conditions given above. This way, an analytical expression for the monomer concentration at the crystal surface (the one determining the polymerization rate) is readily obtained:

$\left[M_{i}\right]=\eta_{i}\left[M_{i}\right]_{m p}-\frac{r_{c r y s}^{2}}{3 D_{i, \mu p}} R_{p, i}\left(1-\frac{1}{\phi}\right)$

where $\phi$ is the microparticle growth factor, defined as:

$\phi=\frac{R_{\mu p}}{r_{c r y s}}$

According to the selected kinetic scheme, the consumption rates of ethylene and hydrogen can be written as follows:

$R_{p, e t h}=[M e][M] \sum_{k=1}^{n s i t e s}\left(k_{i n}^{k} P_{0}^{k}+k_{p}^{k} \lambda_{0}^{k}+k_{t M}^{k} \lambda_{0}^{k}\right)$

$R_{p, h y d}=[\mathrm{Me}]\left[\mathrm{H}_{2}\right] \sum_{k=1}^{n s i t e s} k_{t H_{2}}^{k} \lambda_{0}^{k}$

where $[\mathrm{Me}]$ is the concentration of active metal in the catalyst, whose value is equal to:

$[M e]=\frac{\omega_{M e} \rho_{c a t}}{M W_{M e}}$ 
where $\omega_{M e}$ is the metal mass fraction in the catalyst, $\rho_{\text {cat }}$ the catalyst density and $M W_{M e}$ the active metal molecular weight. The microparticle growth factor defined above can be conveniently computed in terms of volumes as follows:

$\phi^{3}=\frac{V_{\mu p}}{V_{\text {crys }}}=\frac{V_{\text {crys }}+V_{p o l}}{V_{\text {crys }}}=1+\frac{[M e] M W_{\text {mon }} \sum_{k=1}^{n s i t e s} \mu_{1}^{k}}{\rho_{\text {pol }}}$

where $V_{\mu p}$ is the microparticle volume, $V_{\text {crys }}$ the catalyst crystal volume, $V_{p o l}$ the polymer shell volume and $\rho_{p o l}$ the polymer density.

Focusing on the macroparticle and again considering spherical geometry, the mass balance for a generic monomer can be written as follows:

$\frac{\partial\left[M_{i}\right]_{m p}}{\partial t}=\frac{1}{r_{m p}^{2}} \frac{\partial}{\partial r_{m p}}\left(D_{i, m p} r_{m p}^{2} \frac{\partial\left[M_{i}\right]_{m p}}{\partial r_{m p}}\right)-R_{v, i}$

where $\left[M_{i}\right]_{m p}$ is the concentration of the $\mathrm{i}$-th specie in the macroparticle, $r_{m p}$ is the macroparticle radial coordinate, $D_{i, m p}$ the $i$-th species diffusion coefficient in the macroparticle, and $R_{v, i}$ the consumption rate of the $i$-th species per unit volume of macroparticle. The corresponding boundary conditions consider profile symmetry in the macroparticle center and mass transport at the macroparticle/solvent interface:

$$
\begin{array}{ll}
r_{m p}=0 & \frac{\partial\left[M_{i}\right]_{m p}}{\partial r_{m p}}=0 \\
r_{m p}=R_{m p} & -\left.D_{i, m p} \frac{\partial\left[M_{i}\right]_{m p}}{\partial r_{m p}}\right|_{r_{m p}=R_{m p}}=k_{c, i}\left(\left[M_{i}\right]_{m p, i n t}-\left[M_{i}\right]_{b}\right)
\end{array}
$$

where $k_{c, i}$ is the mass transfer coefficient of the $i$-th species, $\left[M_{i}\right]_{m p \text {,int }}$ the concentration of the $i$-th species at the polymer macroparticle/solvent interface, and $\left[M_{i}\right]_{b}$ the concentration of the $i$-th species in the surrounding environment. All mass transfer coefficients can be estimated through the Ranz-Marshall relation ${ }^{17}$. The microparticle consumption rate $R_{p, i}$ is related to the macroparticle consumption rate $R_{v, i}$ through the following expression:

$R_{v, i}=R_{p, i}\left(\frac{1-\varepsilon}{\phi^{3}}\right)$

where $\varepsilon$ is the local void fraction of the macroparticle.

Finally, it is worth noticing that the diffusion coefficient which appears in eq. 10 depends on the catalyst void fraction and tortuosity ${ }^{[9,10,21,42]}$, as commonly assumed in heterogeneous catalysis: 
$D_{i, m p}=D_{i, b} \frac{\varepsilon}{\tau}$

where $D_{i, b}$ is the diffusion coefficient of the $i$-th species in the surrounding solvent and $\tau$ the catalyst tortuosity. Consistently with the approach proposed by Hutchinson and coworkers ${ }^{[10]}$, it is here assumed that the macroparticle void fraction varies in time and space because of the uneven grow rate within the particle. Accordingly, combining eq. 10 and eq. 14, and after some manipulations, the macroparticle mass balance can be re-written as follows:

$\frac{\partial\left[M_{i}\right]_{m p}}{\partial t}=\frac{D_{i, b}}{\tau}\left(\frac{2 \varepsilon}{r_{m p}} \frac{\partial\left[M_{i}\right]_{m p}}{\partial r_{m p}}+\varepsilon \frac{\partial^{2}\left[M_{i}\right]_{m p}}{\partial r_{m p}^{2}}+\frac{\partial \varepsilon}{\partial r_{m p}} \frac{\partial\left[M_{i}\right]_{m p}}{\partial r_{m p}}\right)-R_{v, i}$

where the following boundary condition is applied for the void fraction:

$r_{m p}=\left.0 \quad \frac{\partial \varepsilon}{\partial r_{m p}}\right|_{r_{m p}=0}=0$

Assuming that the spatial arrangement of the microparticles does not change during polymerization ${ }^{13}$, the volume growth depends only on the local growth factor. For every generic radial position, the following volume balance can be written at every time instant $t$ :

$V_{s}(t=0) \phi^{3}(t)(1-\varepsilon(t=0))=V_{s}(t)(1-\varepsilon(t))$

This equation allows computing the void fraction for every radial position and time instant. According to the aforementioned assumption, it is also possible to account for the time evolution of the spherical shell in a generic radial position:

$\frac{\partial V_{S}}{\partial t}=V_{S}(t=0) \frac{\partial \phi^{3}}{\partial t}$
$\frac{\partial \phi^{3}}{\partial t}=\frac{[M e] M W_{\text {mon }}}{\rho_{\text {pol }}} \sum_{k=1}^{n \text { sites }} \frac{\partial \mu_{1}^{k}}{\partial t}$

Additional balances are required in order to describe the activation dynamics of the catalyst active sites by the cocatalyst and the spontaneous deactivation:

$$
\begin{aligned}
& \frac{\partial P_{*}^{k}}{\partial t}=k_{\text {act }}^{k} C_{p}^{k}[\text { cocat }]-k_{\text {deact }}^{k} P_{\text {tot }}^{k} \\
& \frac{\partial C_{d}^{k}}{\partial t}=k_{\text {deact }}^{k} P_{\text {tot }}^{k}
\end{aligned}
$$


where [cocat] is the cocatalyst concentration, $P_{t o t}{ }^{k}$ is the sum of vacant and occupied active sites of type $k$ and $C_{d}{ }^{k}$ the amount of deactivated sites of type $k$. The fraction of active sites of type $k$ not yet activated, $C_{p}{ }^{k}$, is defined as follows:

$C_{p}^{k}=C_{p 0}^{k}-C_{d}^{k}-P_{t o t}^{k}$

where $C_{p 0}{ }^{k}$ is the fraction of active metal which leads to active sites of type $k$. This quantity is particularly relevant since it determines the activity of the catalyst.

The concentration of a few additional species are needed to complete the single-particle model. Namely, the concentration of the vacant sites of type $k, P_{0}{ }^{k}$, is calculated as follows:

$P_{0}^{k}=P_{t o t}^{k}-\lambda_{0}^{k}$

All computational details about model development, omitted here for the sake of brevity, are reported in the Supporting Information.

\section{Modeling of the polymer molecular weight}

The population balances required to evaluate the molecular weight distributions are written in the generic radial position inside the spherical macroparticle. Since we focus on average properties rather than on the complete molecular weight distribution, the method of moments is applied to reduce the computational effort $^{[11]}$. The $l$-th order moment relative to the polymer chains growing on an active site of type $k$ is defined as follows:

$\lambda_{l}^{k}=\sum_{n=1}^{\infty} n^{l} P_{n}^{k}$

By applying this definition to the population balance of the growing chains, the following equations for the moments of the first three orders are obtained for each active site of type $k$ :

$\frac{\partial \lambda_{0}^{k}}{\partial t}=[M]\left(k_{t M}^{k} \lambda_{0}^{k}+k_{\text {in }}^{k} P_{0}^{k}\right)-\lambda_{0}^{k}\left(k_{\text {deact }}^{k}+k_{t M}^{k}[M]+k_{t H}^{k}\left[H_{2}\right]+k_{p}^{k}[M]\right)+k_{p}^{k}[M] \lambda_{0}^{k}$
$\frac{\partial \lambda_{1}^{k}}{\partial t}=[M]\left(k_{t M}^{k} \lambda_{0}^{k}+k_{\text {in }}^{k} P_{0}^{k}\right)-\lambda_{1}^{k}\left(k_{\text {deact }}^{k}+k_{t M}^{k}[M]+k_{t H}^{k}\left[H_{2}\right]+k_{p}^{k}[M]\right)+k_{p}^{k}[M]\left(\lambda_{0}^{k}+\lambda_{1}^{k}\right)$
$\frac{\partial \lambda_{2}^{k}}{\partial t}=[M]\left(k_{t M}^{k} \lambda_{0}^{k}+k_{\text {in }}^{k} P_{0}^{k}\right)-\lambda_{2}^{k}\left(k_{\text {deact }}^{k}+k_{t M}^{k}[M]+k_{t H}^{k}\left[H_{2}\right]+k_{p}^{k}[M]\right)+k_{p}^{k}[M]\left(\lambda_{0}^{k}+2 \lambda_{1}^{k}+\lambda_{2}^{k}\right)$

The partial derivative on the left side is due to the fact that the reaction rate varies not only in time but also along macroparticle radius $r_{m p}$, because of the non-uniform monomer concentration profile within the growing particle as a consequence of the interplay between reactions and diffusion phenomena (vide infra). 
The overall polymer (defined as the sum of living and dead chains) is similarly characterized by the corresponding population balance and once more applying the method of moments. The $l$-th order moment for the overall polymer produced at the active site of type $k$ is defined as:

$\mu_{l}^{k}=\lambda_{l}^{k}+\sum_{n=1}^{\infty} n^{l} D_{n}^{k}$

After some manipulations, combining eq. 28 with the population balance for the bulk polymer, the following set of equations are obtained for each active site:

$\frac{\partial \mu_{0}^{k}}{\partial t}=k_{t M}^{k}[M] \lambda_{0}^{k}+k_{i n}^{k}[M] P_{0}^{k}$

$\frac{\partial \mu_{1}^{k}}{\partial t}=\frac{\partial \mu_{0}^{k}}{\partial t}+k_{p}^{k}[M] \lambda_{0}^{k}$

$\frac{\partial \mu_{2}^{k}}{\partial t}=\frac{\partial \mu_{0}^{k}}{\partial t}+k_{p}^{k}[M]\left(\lambda_{0}^{k}+2 \lambda_{1}^{k}\right)$

The average properties of the cumulative molecular weight distributions are readily evaluated from these moments; namely, the main average molecular weights and polydispersity are expressed as follows:

$M W_{n}=M W_{m o n} \frac{\sum_{k=1}^{n s i t e s} \mu_{1}^{k}}{\sum_{k=1}^{n s i t e s} \mu_{0}^{k}}$

$M W_{w}=M W_{m o n} \frac{\sum_{k=1}^{n s i t e s} \mu_{2}^{k}}{\sum_{k=1}^{n \text { nites }} \mu_{1}^{k}}$

$P D=\frac{\sum_{k=1}^{n s i t e s} \mu_{2}^{k} \sum_{k=1}^{n s i t e s} \mu_{0}^{k}}{\left(\sum_{k=1}^{n s i t e s} \mu_{k}^{1}\right)^{2}}$

where $M W_{n}$ is the number average molecular weight, $M W_{w}$ the weight average molecular weight, $P D$ the polymer polydispersity, and $M W_{\text {mon }}$ the monomer molecular weight. All these quantities correspond to a generic microparticle, then to a generic radial position inside the macroparticle. The evaluation of the average quantities for a macroparticle is performed through an integral average of the moments over macroparticle volume:

$\mu_{l, a v e}(t)=\frac{1}{V_{m p}(t)} \int_{r_{m p=0}}^{r_{m p}=R_{m p}} 4 \pi r_{m p}^{2} \sum_{k=1}^{n s i t e s} \mu_{l}^{k}\left(r_{m p}, t\right) d r_{m p}$

where $\mu_{1, \text { ave }}$ is the average $l$-th order moment and $V_{\mathrm{mp}}$ is the macroparticle volume. The number average and weight average molecular weights and polydispersity for the entire particle can be computed as follows:

$M W_{n, \text { ave }}=\frac{\mu_{1, \text { ave }}}{\mu_{0, a v e}} M W_{\text {mon }}$ 
$M W_{w, \text { ave }}=\frac{\mu_{2, \text { ave }}}{\mu_{1, \text { ave }}} M W_{\text {mon }}$

$P D_{\text {ave }}=\frac{M W_{w, \text { ave }}}{M W_{n, \text { ave }}}$

\section{Numerical solution - single-particle model}

The numerical solution of the multigrain model (i.e., macroparticle mass balances and moment equations) has been performed by discretizing the macroparticle radial coordinate in $j$ grid points and applying the method of lines ${ }^{[43]}$, which allows obtaining a system of ordinary differential equations (ODE) where time is the only independent variable. In particular, discretized mass balances of ethylene, hydrogen and active/deactivated catalyst sites, as well as moment equations, are written in each discretization point.

As mentioned above, the macroparticle volume increases because of the polymerization reaction. The uneven monomer profile within the particle implies that each spherical shell grows with its own kinetics, and this also modifies the local void fraction. To properly account for this evolution, the void fraction in the $j$-th grid point is computed at each instant time through the following volume balance:

$V_{j, 0} \phi_{j}^{3}=\frac{4}{3} \pi\left(r_{m p, j}^{3}-r_{m p, j-1}^{3}\right)\left(1-\varepsilon_{j}\right)$
$V_{j, 0}=\frac{4}{3} \pi\left(r_{m p, j, 0}^{3}-r_{m p, j-1,0}^{3}\right)\left(1-\varepsilon_{j, 0}\right)$

where $V_{j, 0}$ is the initial volume of the spherical shell in the $j$-th grid point (defined by eq. 40), $\varepsilon_{j}$ and $\varepsilon_{j, 0}$ are actual and initial void fraction in the same grid point, and $r_{m p, j, 0}$ is the initial position of the $j$-th point along the discretized radial coordinate. However, the implementation of eq. 39 alone is not sufficient to compute the local void fraction, since $r_{j}$ values are still unknown. Assuming that the spatial arrangement of the microparticles does not change ${ }^{[10]}$, the position of the $j$-th grid point at every time instant depends only on initial position and local growth factor through the following relationship:

$r_{m p, j}=r_{m p, j, 0} \phi_{j}$

Local void fraction can thus be computed as a function of the integration variables combining eqs. 39, 40 and 41.

Finally, a remark about the approach used to account for the variable particle volume is needed. Even though the particle size is obviously changing in time, all the equations above apply to constant particle volume. This inconsistency is handled by adopting a discretized mass balance for each generic grid point written in terms of number of moles. This equation is readily obtained starting from the differentiation of the definition of number of moles in a generic spherical shell in the $j$-th grid point:

$\frac{d N_{i, j}}{d t}=\frac{d\left(M_{i, j} V_{j}\right)}{d t}=M_{i, j} \frac{d V_{j}}{d t}+V_{j} \frac{d M_{i, j}}{d t}$ 
$V_{j}=\frac{4}{3} \pi\left(r_{m p, j, 0}^{3} \phi_{j}^{3}-r_{m p, j-1,0}^{3} \phi_{j-1}^{3}\right)$

where $N_{i, j}$ and $M_{i, j}$ are the number of moles and the concentration of the $i$-th specie in the $j$-th spherical shell, respectively, and $V_{j}$ is the volume of the $j$-th spherical shell. According to this definition, and taking advantage of eq. 15 , the discretized mass balance for the monomer generic $j$-th grid point can be written as follows:

$\frac{1}{V_{j}} \frac{d N_{i, m p, j}}{d t}=\frac{D_{i, b}}{\tau}\left(\frac{2 \varepsilon_{j}}{r_{m p, j}} \frac{d\left[M_{i}\right]_{m p, j}}{d r_{m p, j}}+\varepsilon_{j} \frac{d^{2}\left[M_{i}\right]_{m p, j}}{d r_{m p, j}^{2}}+\frac{d \varepsilon_{j}}{d r_{m p, j}} \frac{d\left[M_{i}\right]_{m p, j}}{d r_{m p, j}}\right)-R_{v, i, j}+\frac{\left[M_{i}\right]_{m p, j}}{V_{j}} \frac{d V_{j}}{d t}$

where the monomer concentration $\left[M_{i}\right]_{m p, j}$ is no longer calculated through eq. 15 but actually evaluated as:

$\left[M_{i}\right]_{m p, j}=\frac{N_{i, m p, j}}{V_{j}}$

Radial derivatives are expressed through a suitable finite differences scheme. The time derivative of each spherical shell can be easily obtained by differentiating the definition of spherical shell while taking advantage of eq. 43:

$\frac{d V_{j}}{d t}=\frac{4}{3} \pi\left(\frac{d \phi_{j}^{3}}{d t} r_{m p, j, 0}^{3}-\frac{d \phi_{j-1}^{3}}{d t} r_{m p, j-1,0}^{3}\right)$

$\frac{d \phi_{j}^{3}}{d t}=\frac{[M e] M W_{\text {mon }}}{\rho_{\text {pol }}} \sum_{k=1}^{n s i t e s} \frac{d \mu_{1, j}^{k}}{d t}$

Finally, it is worth mentioning that, $V_{j}$ is intended as the volume of macroparticle shell, which includes the catalyst, the polymer and the liquid-filled pores (that is, the voids among the microparticles). In this framework, the positions of the radial grid points are automatically updated at each integration step through eq. 41 . Moreover, the use of an integration algorithm for stiff ODE systems allows to adequately track the initial rapid volume growth and to increase the time step during the final stage of polymerization, when monomer consumption is slower because of spontaneous catalyst deactivation.

\section{Modeling of the slurry semibatch reactor}

Mass balances on both liquid and gas phases have been considered. For the gas phase, the mass balances are written in terms of overall number of moles:

$\frac{d N_{i}}{d t}=Q_{i}-k_{l, i} S_{i n t}\left(\left[M_{i}\right]_{b, e q}-\left[M_{i}\right]_{b}\right)$

where $N_{i}$ is the number of moles of the $i$-th species in gas phase, $Q_{i}$ the molar inlet flow of the $i$-th species in gas phase, $k_{l, i}$ the gas/liquid mass transfer coefficient related to the $i$-th species, $S_{\text {int }}$ the gas/liquid interface, and $\left[M_{i}\right]_{b, e q}$ the equilibrium concentration of the $i$-th species in liquid phase. Note that mass transfer resistance 
located in liquid phase is assumed in eq. 48 , i.e. the mass transfer coefficient is equal to the one in the liquid phase. The inlet flow $Q_{i}$ is calculated so that the partial pressure in the gas phase of each component is constant, in agreement with the experimental setup. Assuming constant pressure and temperature, the ideal gas law of each component is differentiated with respect to time:

$P_{i} \frac{d V_{g}}{d t}=R T \frac{d N_{i}}{d t}$

where $P_{i}$ is the partial pressure of the $i$-th species, $V_{g}$ the volume of the gas phase, $R$ the universal gas constant, and $T$ the absolute temperature. The total reactor volume can be expressed as the sum of three different contributions:

$V_{\text {react }}=V_{g}+V_{l}+V_{p}$

where $V_{l}$ is the volume of the liquid phase (that is, the solvent) and $V_{p}$ the overall solid volume. Since total reactor and solvent volumes are constant during the reaction, the following expression can be obtained:

$\frac{d V_{\text {react }}}{d t}=\frac{d V_{g}}{d t}+\frac{d V_{p}}{d t}=0$

As expected, eq. 51 states that the decreasing rate of gas phase volume is equal to the overall solid growth rate. Moreover, an analytical expression for solid growth rate is obtained from eq. 46:

$\frac{d V_{p}}{d t}=\left.N_{\text {part }} \frac{4}{3} \pi R_{m p, 0}^{3} \frac{d \phi^{3}}{d t}\right|_{r_{m p}=R_{m p, 0}}=N_{\text {part }} \sum_{j=1}^{n g r i d} \frac{d V_{j}}{d t}$

where $N_{\text {part }}$ is the overall number of macroparticles in the slurry. Assuming negligible particle breakage and aggregation inside the reactor, this value is assumed to be constant and equal to the initial number of catalyst particles fed to the system. Finally, the molar inlet flow is expressed combining eqs. 49, 51, and 52 as:

$Q_{i}=k_{l, i} S_{i n t}\left(\left[M_{i}\right]_{b, e q}-\left[M_{i}\right]_{b}\right)-\frac{N_{i}}{V_{g}} \frac{d V_{p}}{d t}$

For the liquid phase, the following mass balances can be written:

$\frac{d\left[M_{i}\right]_{b}}{d t}=k_{l, i} \frac{s_{\text {int }}}{V_{l}+V_{p}}\left(\left[M_{i}\right]_{b, e q}-\left[M_{i}\right]_{b}\right)-N_{\text {part }} \frac{s_{p}}{V_{l}} k_{c, i}\left(\left[M_{i}\right]_{b}-\left[M_{i}\right]_{m p, i n t}\right)$

where $S_{p}$ is the macroparticle surface, that is, the interface between solid and liquid phases. 
In the previous equations, the transport terms involve the solubility of each species in the liquid phase, which have been evaluated through thermodynamic equilibrium relationships assuming ideal behavior (i.e., Henry and Raoult laws):

$P_{i}=H_{i}(T) x_{i} \quad i=$ ethylene, hydrogen

$P_{i}=P_{i}^{0}(T) x_{i} \quad i=$ n-hexane

\section{Numerical solution - overall model}

The overall model is then constituted by the multigrain model, aimed at describing particle growth and morphology, combined with the mass balances needed to characterize the behavior of the semibatch reactor. The complete set of equations described above is summarized in the Supporting Information. As mentioned above, the multigrain model can be reduced to a system of ODE by discretizing the macroparticle radial coordinate and applying the method of lines. The final system of equations, including the reactor balances, is then a large set of ODE, to be numerically solved. Such ODE system was numerically integrated by means of ode15s algorithm as implemented in MATLAB. Considering that two species are present in the system (ethylene and hydrogen) and that two kinds of catalytic active sites are considered, the overall number of ODE is equal to $(19 n p+4)$, where $n p$ is the number of discretization points along the macroparticle radial coordinate. Typically, $n p$ values ranging from 50 to 150 have been used. Initial conditions are reported in the Supporting Information.

The closure of the mass balances was checked for both the single particle model and the overall particle/reactor system by means of two error functions, which provide an estimation of the inaccuracy due to the discretization as a function of the number of grid points (more details are available in the Supporting Information). Data fitting was performed by means of the genetic algorithm as implemented in MATLAB (GDE3 function), by minimizing the sum of the square of the errors as objective function:

$F=\sum_{i=1}^{n_{\exp }}\left(y_{i}-y_{i}^{e x p}\right)^{2}$

where $F$ is the objective function, $n_{\exp }$ is the number of experimental points, $y_{i}^{\text {exp }}$ is the i-th experimental point and $y_{i}$ is the corresponding model prediction. No weights have been introduced in order to account for the different experimental errors related to number average and weight average molecular weights.

\section{Input data and parameter evaluation}

All used input data are summarized in Table 2. The particle diameter is normalized with respect to its initial value (that is, the normalized catalyst particle diameter is equal to 1 ). 


\begin{tabular}{ccccc}
\hline & & & Ethylene & Hydrogen \\
\hline $\mathrm{d}_{\mathrm{cat}}[-]$ & 1 & $\mathrm{D}_{\mathrm{mp}}\left[\mathrm{cm}^{2} \mathrm{~s}^{-1}\right]$ & $1.1 \cdot 10^{-4}$ & $1 \cdot 10^{-3}$ \\
$\mathrm{~d}_{\text {crys }}[\mu \mathrm{m}]$ & 0.01 & $\mathrm{D}_{\mu \mathrm{p}}\left[\mathrm{cm}^{2} \mathrm{~s}^{-1}\right]$ & $1.6 \cdot 10^{-6}$ & $1 \cdot 10^{-5}$ \\
$\rho_{\text {cat }}\left[\mathrm{g} \mathrm{cm}^{-3}\right]$ & 2.84 & $\eta[-]$ & 0.05 & 0.12 \\
$\omega_{\text {Me }}[-]$ & 0.11 & $\mathrm{k}_{1}\left[\mathrm{~cm} \mathrm{~s}^{-1}\right]$ & 10 & 10 \\
$\varepsilon_{\text {in }}[-]$ & 0.25 & & & \\
$\tau[-]$ & 6 & & & \\
$\rho_{\text {pol }}\left[\mathrm{g} \mathrm{cm}^{-3}\right]$ & 0.96 & & & \\
\hline
\end{tabular}

Table 2. Model input data.

Diffusion coefficients of ethylene and hydrogen for both micro- and macroparticle, initial catalyst porosity and tortuosity were taken from literature ${ }^{[10]}$. The partition factors $\eta_{i}$ (which determine the monomer concentration at the catalyst surface, as shown in eq. 4) were evaluated from SABIC internal data. Liquid/solid mass transfer coefficients were estimated through Ranz-Marshall equation ${ }^{[44]}$, while gas/liquid mass transfer coefficients were set large enough not to provide any limitation to the polymerization process, in agreement with SABIC internal data. Henry coefficients for ethylene and hydrogen, and n-hexane vapor pressure were taken from Neto et al. ${ }^{[20]}$.

All kinetic constants, as well as the fraction of active metal which leads to an active site of type $\mathrm{k} C p_{0}{ }^{k}$ (which appears in eq. 22) were estimated by direct fitting of the model predictions to experimental data; all considered experimental runs are summarized in Table 3.

\begin{tabular}{|c|c|c|c|c|c|c|c|}
\hline Data set & $\mathbf{P}$ [bar] & $\mathbf{P}_{\mathrm{C} 2}[\mathrm{bar}]$ & $\mathbf{P}_{\mathrm{H} 2}$ [bar] & $\mathbf{T}[\mathrm{C}]$ & $\begin{array}{c}\text { cocat } \\
{\left[\mathrm{mol} \mathrm{cm}^{-3}\right]}\end{array}$ & $\mathbf{m}_{\text {cat }}[\mathrm{mg}]$ & $t$ [min] \\
\hline 1 & 1.83 & 1.1 & 0 & 85 & $0.8 \cdot 10^{-6}$ & 20 & 120 \\
\hline 2 & 2.38 & 1.1 & 0.55 & 85 & $0.8 \cdot 10^{-6}$ & 60 & 120 \\
\hline 3 & 2.93 & 1.1 & 1.1 & 85 & $0.8 \cdot 10^{-6}$ & 60 & 120 \\
\hline 4 & 4.03 & 1.1 & 2.2 & 85 & $0.8 \cdot 10^{-6}$ & 60 & 120 \\
\hline 5 & 7.33 & 1.1 & 5.5 & 85 & $0.8 \cdot 10^{-6}$ & 60 & $60 / 120$ \\
\hline
\end{tabular}

Table 3. Experimental data sets used for model fitting and corresponding experimental conditions.

The total fraction of catalytic sites which can be activated is defined as the sum of the contribution of each kind of catalytic site:

$C_{p 0}=C_{p 0}^{1}+C_{p 0}^{2}=\alpha C_{p 0}+(1-\alpha) C_{p 0}$

where $C_{p 0}$ is the overall fraction of active metal (cf. eq(22)) and $\alpha$ is the fraction of active sites of type 1. 
Summarizing, the fitting was performed to estimate $C_{p 0}, \alpha$, and the kinetic constants, employing the five experimental runs in the table. In particular, the kinetic parameters were determined in order to best reproduce the inlet ethylene flow (i.e., ethylene propagation rate) and volume-average molecular weights defined by eq. 37-38 as a function of time.

As indicated by experimental data, hydrogen decreases catalyst activity. This inhibition effect is taken into account assuming that the maximum fraction of active sites which can be activated, $C_{p 0}$, decreases at increasing hydrogen partial pressure in the system according to an empirical law reproducing the reversible adsorption mechanism. Moreover, it is assumed that such adsorption is instantaneous, because of the fast hydrogen diffusion within the catalyst; in other words, hydrogen adsorption is fully occurred before the onset of any reaction. On the other hand, the same inhibition is considered reversible, because experimental data showed that catalyst activity can be partially recovered by flashing the slurry after the first reaction step. The validity of this assumption is discussed in detail in the following section (vide infra).

For most data sets, polymer properties are available only at one time point, i.e., after 2 hours. However, for those experiments where more time points are available (data set 5 in Table 3 and data set 9 in Table 8), the average molecular weights remain practically constant already after the first hour of polymerization. Therefore, the fitting procedure has been carried out assuming average molecular weights after one hour of reaction constant and equal to the experimental values measured after two hours. The remaining experimental results were simulated using only the fraction of active metal $C_{p 0}$ as adjustable parameter so that hydrogen inhibiting effect can be accounted for, while the values of $\alpha$ and kinetic constants were kept unchanged.

The estimated values of the kinetic constants are summarized in Table 4. Note that dimensionless values of the propagation and chain transfer rate constants have been reported, as normalized with respect to the propagation constant of site $1 \mathrm{k}_{\mathrm{p}}{ }^{1}$; on the other hand, activation and deactivation constants are reported as absolute values with dimensions. The values of overall fraction of active metal and of fraction of active sites of type 1 as a function of the hydrogen partial pressure are listed in Table 5.

\begin{tabular}{ccc}
\hline Kinetic constant & Site 1 & Site 2 \\
\hline $\mathrm{k}_{\mathrm{act}}{ }^{\mathrm{k}}\left[\mathrm{s}^{-1}\right]$ & $6.17 \cdot 10^{4}$ & $8.50 \cdot 10^{3}$ \\
$\mathrm{k}_{\mathrm{p}} \mathrm{k} / \mathrm{k}_{\mathrm{p}}{ }^{1}[-]$ & 1 & 0.15 \\
$\mathrm{k}_{\mathrm{tM}}{ }^{\mathrm{k}} / \mathrm{k}_{\mathrm{p}}{ }^{1}[-]$ & $5.34 \cdot 10^{-5}$ & $1.02 \cdot 10^{-4}$ \\
$\mathrm{k}_{\mathrm{tH}}{ }^{\mathrm{k}} / \mathrm{k}_{\mathrm{p}}{ }^{1}[-]$ & $1.60 \cdot 10^{-3}$ & $4.40 \cdot 10^{-3}$ \\
$\mathrm{k}_{\text {deact }}{ }^{\mathrm{k}}\left[\mathrm{s}^{-1}\right]$ & $1.41 \cdot 10^{-4}$ & $1.41 \cdot 10^{-4}$
\end{tabular}

Table 4. Kinetic constants. Propagation and chain transfer constants have been normalized with respect to propagation constant of site $1 \mathrm{k}_{\mathrm{p}}{ }^{1}$. 


\begin{tabular}{ccc}
\hline $\mathbf{P}_{\mathbf{H} 2}$ [bar] & $\mathbf{C}_{\mathbf{p 0}}[-]$ & $\boldsymbol{\alpha}[-]$ \\
\hline 0 & 1 & 0.45 \\
0.55 & 0.48 & 0.39 \\
1.1 & 0.37 & 0.39 \\
2.2 & 0.29 & 0.39 \\
5.5 & 0.25 & 0.39
\end{tabular}

Table 5. Overall fraction of active metal and corresponding fraction of active sites as function of hydrogen partial pressure. 


\section{Results and discussion}

\section{Polyethylene synthesis without hydrogen}

The first experimental run involves the polyethylene synthesis carried out without adding hydrogen in the reactor (data set 1 in Table 3). Since hydrogen deactivates significantly the catalyst, this data set is representative of the maximum catalyst activity. For this reason, number average and weight average molecular weights are hereafter expressed as dimensionless values normalized with respect to the experimental values of this specific data set:

$$
\begin{aligned}
M W_{n, \text { ave }}^{\text {norm }}(t) & =\frac{M W_{n, \text { ave }}(t)}{M W_{n, \text { ave }}\left(P_{H 2}=0\right)} \\
M W_{w, \text { ave }}^{\text {norm }}(t) & =\frac{M W_{w, \text { ave }(t)}}{M W_{w, \text { ave }}\left(P_{H 2}=0\right)}
\end{aligned}
$$

where $M W_{n, a v e}\left(P_{H 2}=0\right)$ and $M W_{w, a v e}\left(P_{H 2}=0\right)$ indicate the final values of the number average and weight average molecular weight, respectively, for this same set of experiments. Moreover, both experimental and calculated values of ethylene inlet flow are hereafter shown normalized with respect to the experimental value of the total amount of fed monomer:

$$
Q_{i, n o r m}(t)=\frac{Q_{i}(t)}{\int_{t=0}^{t=t} p_{\text {exp }}(t) d t} \quad i=\text { experimental, model }
$$

where $t_{p o l}$ is the overall duration of the synthesis.

The comparison between the experimental and calculated normalized flows for data set 1 is shown in Figure 1a; the generally good agreement supports the assumed two site kinetic model, which is then an effective compromise between simplicity and accuracy. In Figure 1b, the time evolution of the normalized particle size is reported and compared with the experimental final size. Notably, the final value of the ratio between final and initial size is about 50 , corresponding to a volume growth factor of the order of $10^{5}$, indeed confirming remarkable catalyst activity.
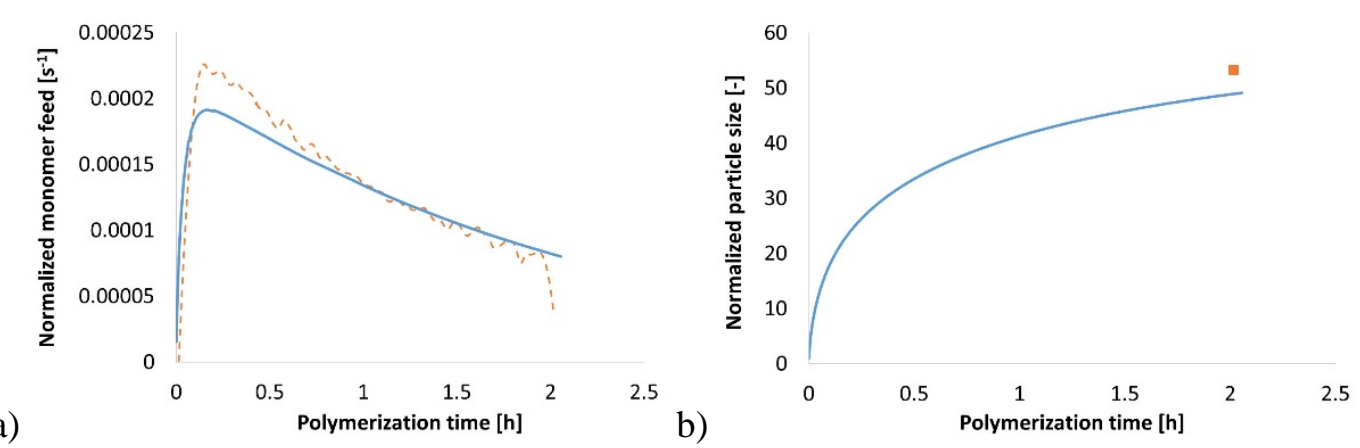

Figure 1. (a) Normalized experimental (dashed line) and calculated ethylene inlet flow as function of time;

(b) normalized experimental (symbol) and calculated particle size as a function of time. 
The time evolutions of the normalized average molecular weights as well as of their ratio (polydispersity) are reported in Figure 2, where the available experimental values are also presented along with the corresponding error bars.

a)

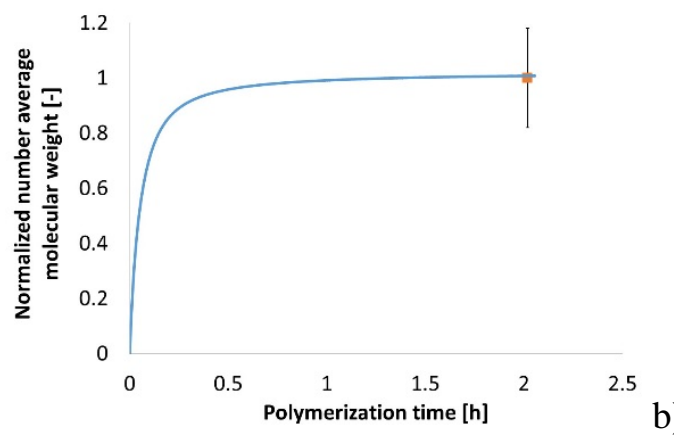

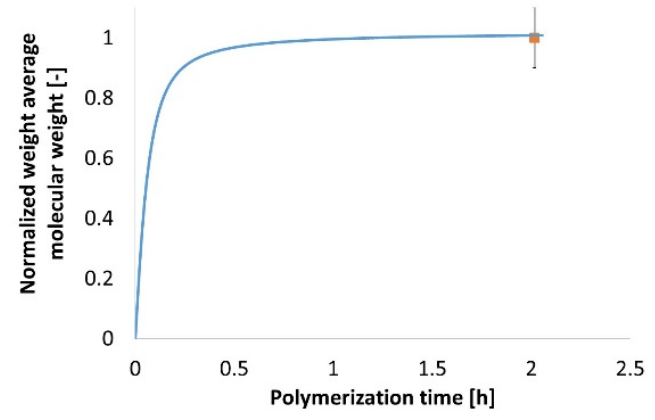

b)

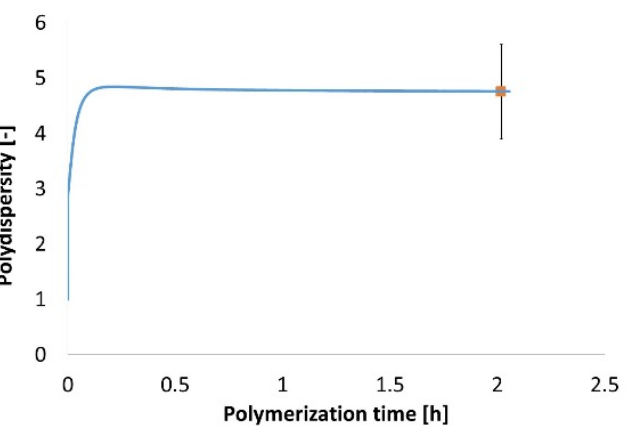

Figure 2. Calculated (continuous lines) and experimental (symbols) normalized number average molecular weight (a), normalized weight average molecular weight (b), and average polydispersity (c) as a function of time.

According to the model, constant values of molecular weight are established after about 30 minutes, thus confirming the quick achievement of stable conditions in terms of polymer microstructure: this behavior can be attributed to the dominant termination mechanisms active without hydrogen, that is, transfer to monomer and spontaneous deactivation. To better support this statement, let us consider the expression of the instantaneous values of number-average degree of polymerization and polydispersity for the polymer chains produced by the catalytic site $k$ :

$D P_{\text {inst }}^{k}=\frac{k_{p}^{k}[M] \lambda_{0}^{k}}{k_{t M}^{k}[M] \lambda_{0}^{k}+k_{\text {deact }}^{k} P_{*}^{k}}$

$P D_{\text {inst }}^{k}=\frac{\frac{\partial \mu_{2}^{k}}{\partial t} \frac{\partial \mu_{0}^{k}}{\partial t}}{\left(\frac{\partial \mu_{1}^{k}}{\partial t}\right)^{2}}$

The volume-averaged values of these same properties are shown as a function of time in Figure 3 for each catalyst site; in particular, dimensionless degrees of polymerization are reported normalized with respect to the value of site 1 . As shown in Figure 3a, the instantaneous degree of polymerization reaches immediately a 
constant value equal to the ratio between the propagation and the transfer rate constants (cf. eq.(62)); moreover, the value of the instantaneous polydispersity in Figure 3b becomes also quickly constant and equal to 2, the instantaneous value corresponding to a system dominated by a monomolecular termination mechanism.
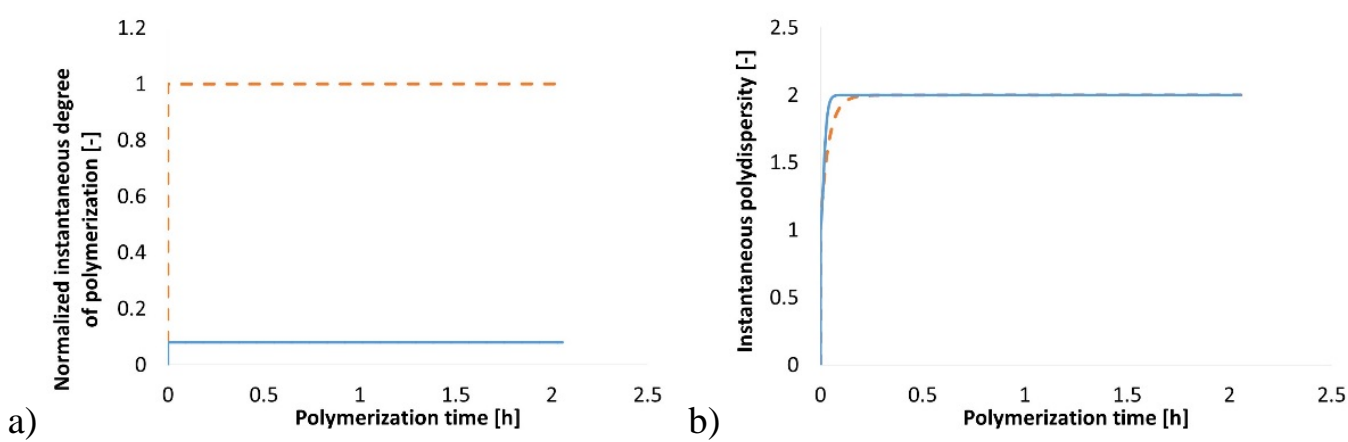

Figure 3. Normalized instantaneous degree of polymerization (a) and instantaneous polydispersity (b) for site 1 and 2 as a function of time.

\section{Polyethylene synthesis with hydrogen}

The effect of hydrogen addition is twofold. On one side, hydrogen reduces catalyst activity by the reversible adsorption mentioned above, while on the other side it acts as chain transfer agent, thus decreasing molecular weights. Catalyst inhibition was accounted for in the model assuming that the fraction of active metal decreases as hydrogen increases, as a consequence of instantaneous hydrogen reversible adsorption. In other words, catalyst activity is reduced right from the beginning of the polymerization and the fraction of active metal is related to that of inhibited active sites, $C p_{H 2}$, as follows:

$C p_{0}+C p_{H_{2}}=1$

Therefore, for each employed set of experimental data, the parameter $C p_{0}$ has been estimated to account for the inhibition of catalyst activity by hydrogen. As shown in Figure 4, the resulting $C p_{\mathrm{H} 2}$ values as a function of the hydrogen partial pressure exhibit a Langmuir type behavior, which is nicely consistent with the assumption of decreasing catalyst activity due to hydrogen adsorption.

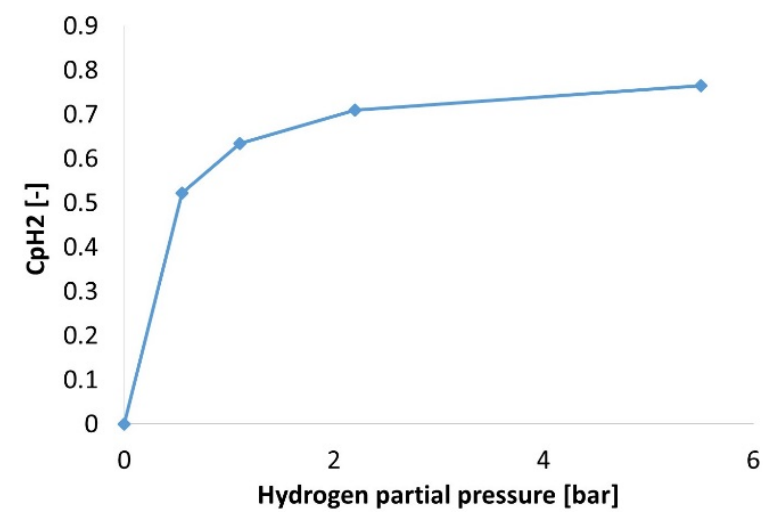


Figure 4. Estimated fraction of inhibited active sites as a function of hydrogen partial pressure for data set 25 in Table 3. The symbols represent the actual values while the line is just intended as eye-guide.

In addition, the relative fraction of active sites $\alpha$ was also assumed to change in the presence of hydrogen, since the inhibition can be at different extent for the two catalytic sites. Actually, the agreement between model and experimental results asked for a slight modification of this quantity only when moving from no-hydrogen to presence of hydrogen; the same parameter value applies at increasing hydrogen partial pressure, as shown in Table 5.

The simulations results are compared with the corresponding experimental data in Table 6 and represented in Figure 5 in terms of final values of the main polymer properties, those available also experimentally. The model provides a generally good description of the main average molecular weights but also of the particle growth rate, given the good agreement in terms of final particle size. For the sake of completeness, the considered experimental size is the $d_{50}$ of the measured final particle size distribution. The complete series of plots for each data set is reported in the Supporting Information.

\begin{tabular}{ccccccccc}
\hline Data set & \multicolumn{2}{c}{$\mathbf{M W}_{\mathbf{n}, \text { ave }}{ }^{\text {norm }}[-]$} & \multicolumn{2}{c}{ MW $_{\text {w,ave }}{ }^{\text {norm }}[-]$} & \multicolumn{2}{c}{ PD,ave $[-]$} & \multicolumn{2}{c}{$\mathbf{d}_{\text {norm }}[-]$} \\
& Exp. & Mod. & Exp. & Mod. & Exp. & Mod. & Exp. & Mod. \\
\hline 1 & 1 & 1 & 1 & 0.97 & 4.7 & 4.6 & 53.3 & 47.8 \\
2 & 0.15 & 0.14 & 0.20 & 0.20 & 6.3 & 6.9 & - & 37.5 \\
3 & $8.16 \cdot 10^{-2}$ & $8.02 \cdot 10^{-2}$ & $1.11 \cdot 10^{-1}$ & $1.19 \cdot 10^{-1}$ & 6.6 & 7 & - & 34.9 \\
4 & $4.91 \cdot 10^{-2}$ & $4.84 \cdot 10^{-2}$ & $7.05 \cdot 10^{-2}$ & $7.14 \cdot 10^{-2}$ & 6.7 & 7 & - & 32.0 \\
5 & $2.81 \cdot 10^{-2}$ & $2.80 \cdot 10^{-2}$ & $4.31 \cdot 10^{-2}$ & $4.02 \cdot 10^{-2}$ & 7.4 & 6.8 & 29.35 & 29.9
\end{tabular}

Table 6. Experimental and calculated normalized values of number average and weight average molecular weights, polydispersity and normalized particle size for the different data sets in Table 3. All values correspond to 2 hours of polymerization time.

a)

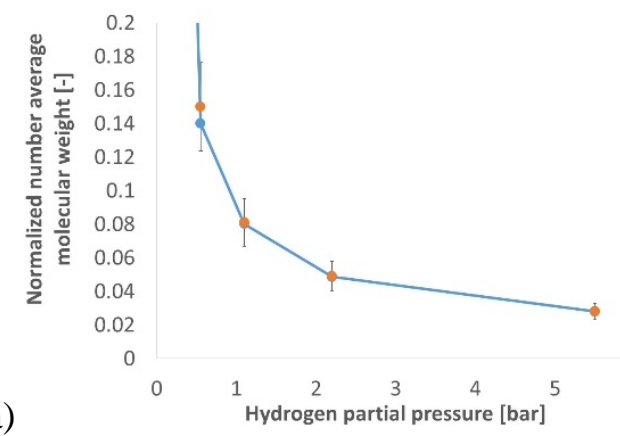

b)

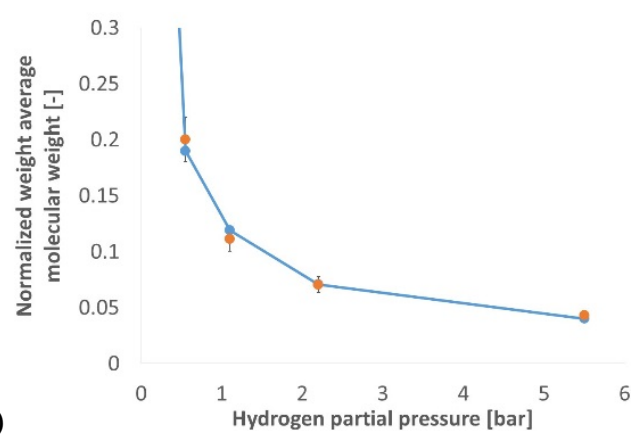



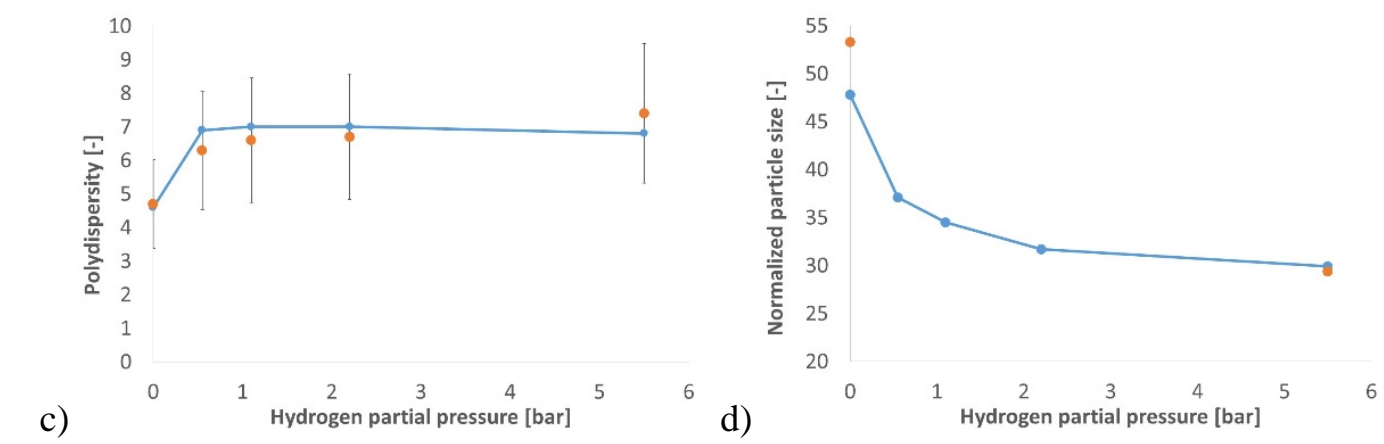

Figure 5. Normalized number average (a) and weight average (b) molecular weights, polydispersity (c) and normalized particle size as a function of hydrogen partial pressure (data sets in Table 3). Symbols: experimental values; lines with symbols: model results.

These results indicate the role of hydrogen as chain transfer agent, since all average molecular weights decrease as hydrogen partial pressure increases. On the other hand, the impact of hydrogen as catalyst inhibitor is better highlighted in terms of catalyst yield (CY), defined as the ratio between the mass of produced polymer and the mass of employed catalyst. Such quantity is shown in Figure 6 as a function of the hydrogen partial pressure; similarly to molecular weights, the values are normalized with respect to the experimental CY of data set 1 . As expected, the normalized CY values decrease as hydrogen partial pressure increases, given the inhibition effect associated with the initial hydrogen adsorption.

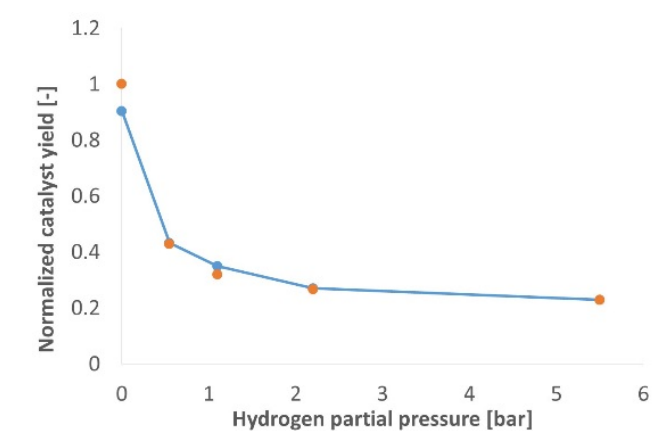

Figure 6. Normalized catalyst yield as a function of hydrogen partial pressure. Symbols: experimental values; lines with symbols: model results.

For data set 5, two set of experimental values are available at two different reaction times, 1 and 2 hours. The comparison between experimental and simulation results confirms the reliability of the predicted time evolution of particle growth and polymer properties, in particular supporting the establishment of almost constant values of the latter properties after very short time. 


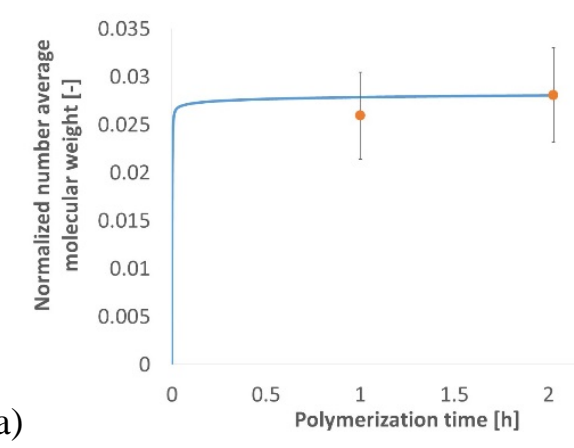

a)

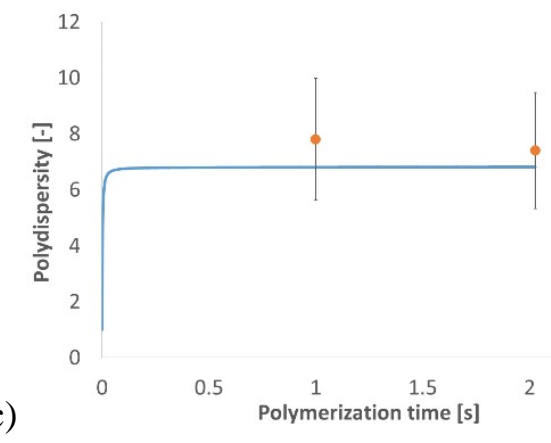

b)

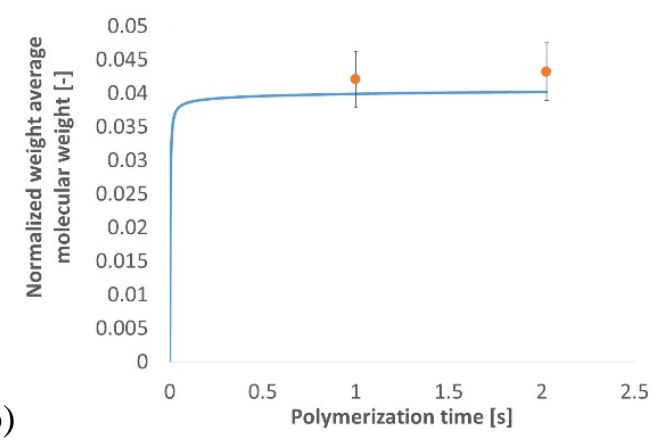

d)

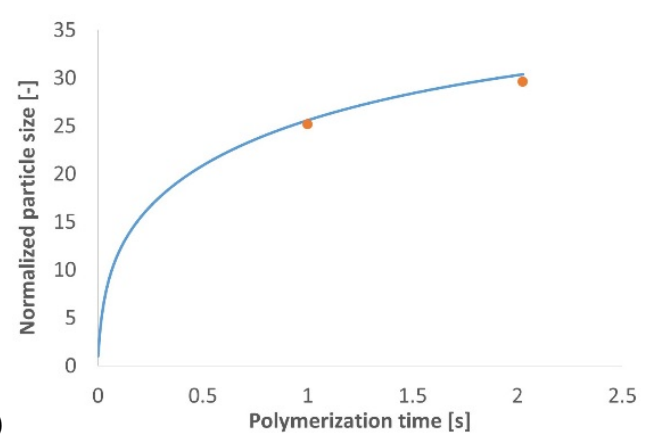

Figure 7. Normalized number average (a) and weight average molecular weight (b), polydispersity (c), and normalized particle size (d) as a function of time for data set 5 in Table 3. Symbols: experimental values; line with symbols: model predictions.

The instantaneous number-average degree of polymerization of the chains produced on the catalytic site $k$ in presence of hydrogen is readily expressed by updating eq.(61) as follows:

$D P_{\text {inst }}^{k}=\frac{k_{p}^{k}[M] \lambda_{0}^{k}}{k_{t M}^{k}[M] \lambda_{0}^{k}+k_{t H}^{k}\left[H_{2}\right] \lambda_{0}^{k}+k_{\text {deact }}^{k} P_{*}^{k}}$

where an additional transfer contribution appears. Therefore, the dominant role of termination by chain transfer becomes even stronger and both instantaneous average degree of polymerization and polydispersity exhibit time evolutions fully equivalent to those shown in Figure 3.

\section{Model validation}

In order to evaluate the general reliability of the model and of the estimated parameter values, model predictions were compared with an independent set of experimental data not employed during the previous model fitting. The reaction conditions of these new experimental runs are summarized in Table 7. Notably, the process conditions differ in terms of monomer and hydrogen partial pressure, and initial amount of catalyst.

\begin{tabular}{cccccccc}
\hline Data set & $\mathbf{P}[$ bar] & $\mathbf{P}_{\mathrm{C} 2}[\mathbf{b a r}]$ & $\mathbf{P}_{\mathrm{H} 2}[\mathbf{b a r}]$ & $\mathbf{T}[\mathbf{C}]$ & $\begin{array}{c}\text { cocat } \\
{\left[\mathbf{m o l ~ c m}^{-3}\right]}\end{array}$ & $\mathbf{m}_{\text {cat }}[\mathbf{m g}]$ & $\mathbf{t}[\mathbf{h r}]$ \\
\hline 6 & 9.13 & 1.4 & 7 & 85 & $0.8 \cdot 10^{-6}$ & 60 & 2
\end{tabular}




$\begin{array}{cccccccc}7 & 7.33 & 1.1 & 5.5 & 85 & 0.8 \cdot 10^{-6} & 20 & 2 \\ 8 & 7.33 & 1.2 & 4 & 85 & 0.8 \cdot 10^{-6} & 30 & 6 \\ 9 & 7.33 & 1.2 & 4 & 85 & 0.8 \cdot 10^{-6} & 50 & 1 / 2 / 5\end{array}$

Table 7. Experimental data sets used for model validation and corresponding experimental conditions.

All simulations have been performed using the same values of all the model parameters estimated before, while the values of $C p_{0}$ were adjusted for each simulation using the hydrogen adsorption isotherm estimated in Figure 4: therefore, a genuinely predictive approach was applied to ensure a meaningful check of the model reliability. The comparison between experimental and simulation results is summarized in Table 8 again in terms of final values of the polymer properties and particle size.

\begin{tabular}{|c|c|c|c|c|c|c|c|c|c|}
\hline \multirow[t]{2}{*}{ Data set } & \multirow[t]{2}{*}{ t [hr] } & \multicolumn{2}{|c|}{ MW $_{\text {n,ave }}{ }^{\text {norm }}[-]$} & \multicolumn{2}{|c|}{ MW $_{\text {w,ave }}{ }^{\text {norm }}[-]$} & \multicolumn{2}{|c|}{$\mathbf{P D}_{\text {,ave }}[-]$} & \multicolumn{2}{|c|}{$\mathbf{d}_{\text {norm }}[-]$} \\
\hline & & Exp. & Mod. & Exp. & Mod. & Exp. & Mod. & Exp. & Mod. \\
\hline 6 & 2 & $2.76 \cdot 10^{-2}$ & $2.99 \cdot 10^{-2}$ & $4.43 \cdot 10^{-2}$ & $4.29 \cdot 10^{-2}$ & 7.7 & 6.8 & - & 34.3 \\
\hline 7 & 2 & $2.70 \cdot 10^{-2}$ & $2.80 \cdot 10^{-2}$ & $3.64 \cdot 10^{-2}$ & $4.01 \cdot 10^{-2}$ & 6.4 & 6.8 & - & 30.3 \\
\hline 8 & 6 & $2.70 \cdot 10^{-2}$ & $3.24 \cdot 10^{-2}$ & $5.23 \cdot 10^{-2}$ & $4.71 \cdot 10^{-2}$ & 9.2 & 6.9 & 36.9 & 37.1 \\
\hline \multirow[t]{3}{*}{9} & 1 & $2.54 \cdot 10^{-2}$ & $3.09 \cdot 10^{-2}$ & $4.77 \cdot 10^{-2}$ & $4.47 \cdot 10^{-2}$ & 9 & 6.9 & 26.9 & 26.2 \\
\hline & 2 & $2.59 \cdot 10^{-2}$ & $3.15 \cdot 10^{-2}$ & $4.77 \cdot 10^{-2}$ & $4.53 \cdot 10^{-2}$ & 8.8 & 6.8 & 30 & 31 \\
\hline & 5 & $2.76 \cdot 10^{-2}$ & $3.20 \cdot 10^{-2}$ & $4.77 \cdot 10^{-2}$ & $4.57 \cdot 10^{-2}$ & 8.2 & 6.8 & 36.3 & 35.3 \\
\hline
\end{tabular}

Table 8. Experimental and calculated values of normalized number average and weight average molecular weights, polydispersity and normalized particle size for data sets in Table 7.

Generally speaking, the agreement between the experimental and model results is satisfactory, especially considering the different durations of the reactions. The comparison between model and simulation results for data set 9 is presented in detail in Figure 8. Some discrepancies appear in terms of number average molecular weights and polydispersity values; moreover, similar discrepancies were found for data set 8 . Since these two experiments have been carried out using a catalyst coming from a different batch from the one used in all other experiments, these discrepancies have been considered acceptable.
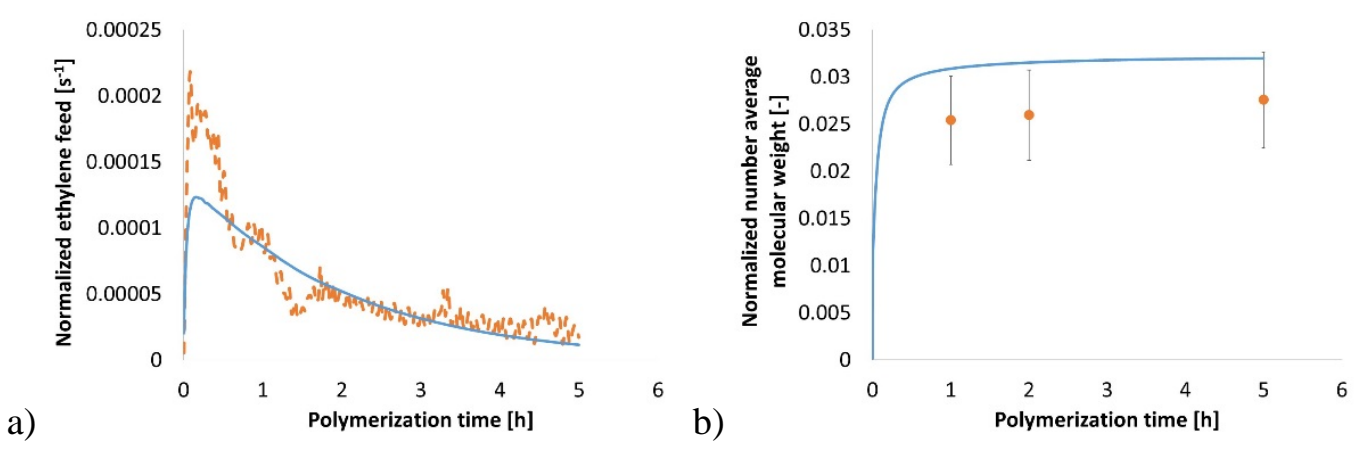

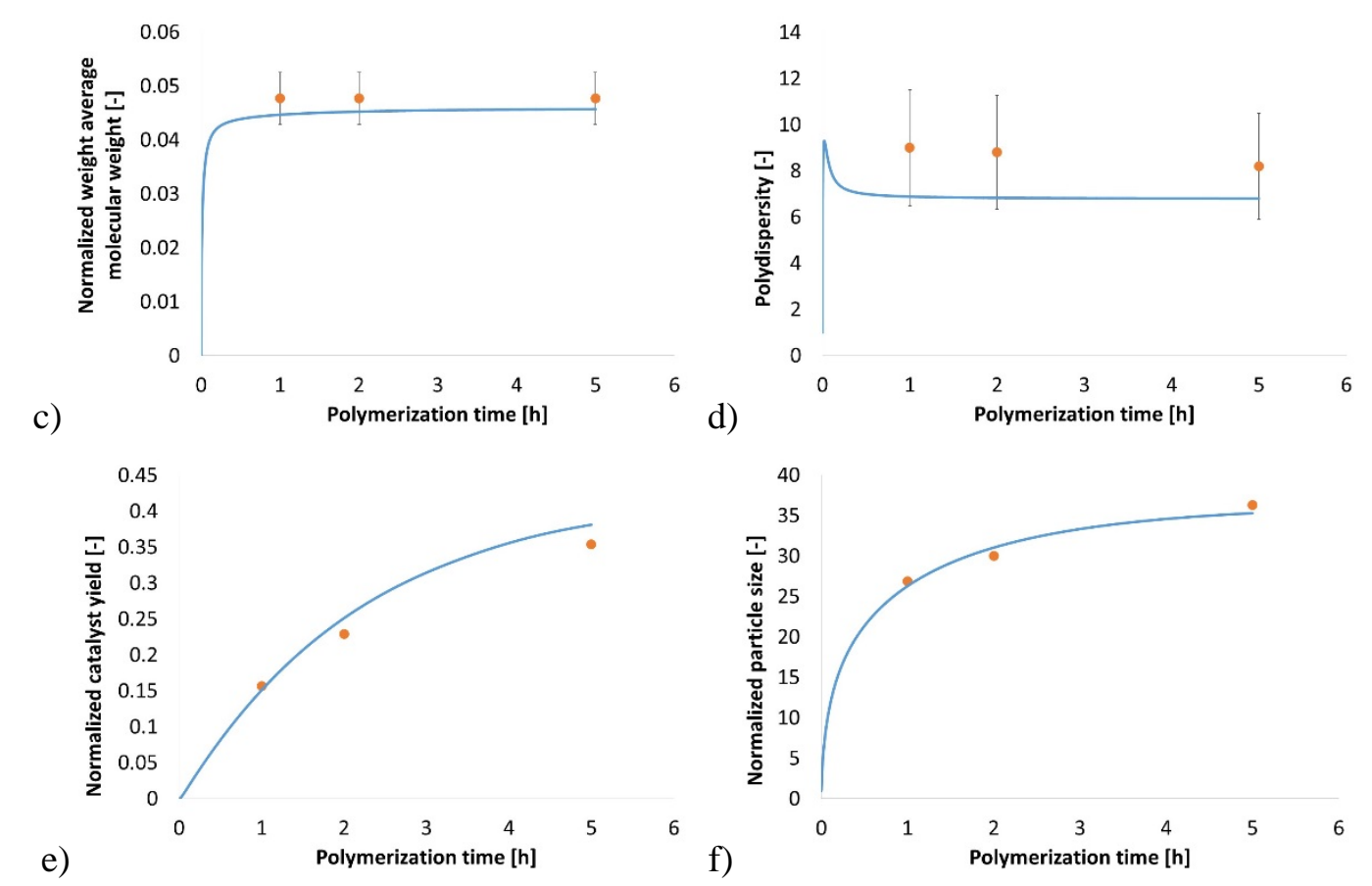

Figure 8. Normalized ethylene inlet flow (a), normalized number average molecular weight (b), normalized weight average molecular weight (c), polydispersity (d), normalized catalyst yield (e) and normalized particle size (f) as a function of polymerization time for data set 9. Dashed lines and dots: experimental values; continuous lines: model results.

\section{Bimodal synthesis}

Model reliability was also tested through an experiment carried out using a fully different operating mode, the so-called "bimodal synthesis", made of two subsequent steps. In the first one, the reaction is performed in presence of hydrogen for 3 hours, while the second step is characterized by the absence of hydrogen and a shorter polymerization time (1 hour). The detailed process conditions are summarized in Table 9. Particle size is not reported since experimental data are not available in this case. As described in the experimental section (vide supra), the reaction is quenched before starting the second step by reducing temperature and pressure, and flushing the reactor with nitrogen. Assuming that catalyst deactivation by hydrogen adsorption is reversible, depressurization and hydrogen removal lead to hydrogen desorption and catalyst regeneration, i.e. to activity recovery. This phenomenon does not affect the catalyst sites spontaneously deactivated during the first step, which remain inactive also during the second step. In the model, this reactivation is accounted for by adjusting the value of $C p_{0}$ at the end of the first step in order to match the experimental ethylene inlet flow (i.e., the initial polymerization rate), while keeping constant the remaining parameter values. Simulations showed that an initial value of potentially active sites $C p_{0}=0.58$ allows to reproduce experimental data, as shown in Figure 9.

\begin{tabular}{lllllccc}
\hline Step & $\mathbf{P}[$ bar $]$ & $\mathbf{P}_{\mathrm{C} 2}[$ bar $]$ & $\mathbf{P}_{\mathrm{H} 2}[$ bar $]$ & $\mathbf{T}[\mathrm{C}]$ & $\begin{array}{c}\text { cocat } \\
{\left[\mathrm{mol} \mathrm{cm}^{-3}\right]}\end{array}$ & $\mathbf{m}_{\text {cat }}[\mathrm{mg}]$ & $\mathbf{t}[\mathrm{hr}]$ \\
\hline
\end{tabular}




\begin{tabular}{llllllll}
1 & 7.33 & 1.1 & 5.5 & 85 & $0.8 \cdot 10^{-6}$ & 60 & 3 \\
2 & 2.13 & 1.4 & 0 & 85 & $0.8 \cdot 10^{-6}$ & 60 & 1 \\
\hline
\end{tabular}

Table 9. Experimental conditions for bimodal synthesis.

\begin{tabular}{cccccccccc}
\hline Step & $\mathbf{t}[\mathrm{hr}]$ & \multicolumn{2}{c}{$\mathbf{M W}_{\mathbf{n} \text {,ave }}{ }^{\text {norm }}[-]$} & \multicolumn{2}{c}{ MW $_{\text {w,ave }}{ }^{\text {norm }}[-]$} & \multicolumn{2}{c}{ PD,ave $[-]$} & \multicolumn{2}{c}{ CY $_{\text {norm }}[-]$} \\
& & Exp. & Mod. & Exp. & Mod. & Exp. & Mod. & Exp. & Mod. \\
\hline 1 & 3 & - & $2.81 \cdot 10^{-2}$ & - & $4.02 \cdot 10^{-2}$ & - & 6.8 & - & 0.34 \\
2 & 1 & $5.41 \cdot 10^{-2}$ & $4.95 \cdot 10^{-2}$ & $4.77 \cdot 10^{-1}$ & $4.54 \cdot 10^{-1}$ & 41.7 & 43.6 & 0.58 & 0.61 \\
\hline
\end{tabular}

Table 10. Experimental and calculated normalized number average and weight average molecular weights, polydispersity and catalyst yield for each step of bimodal synthesis.

a)
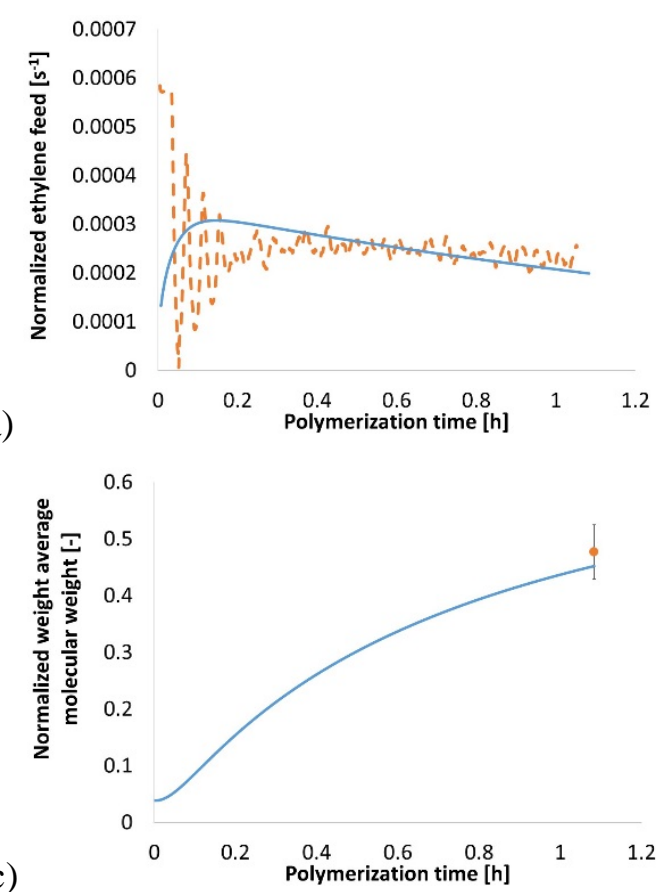

b)
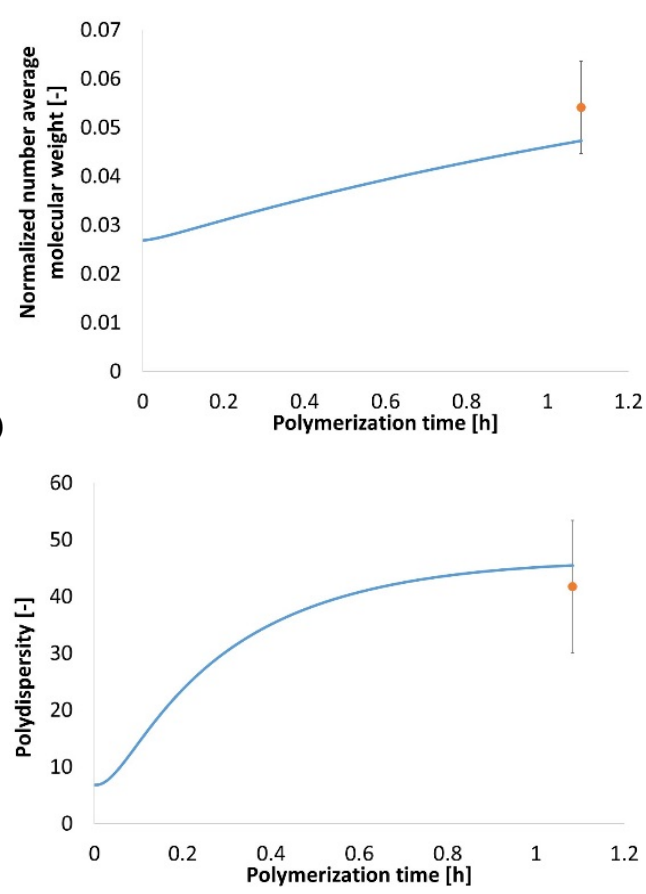

Figure 9. Normalized ethylene inlet flow (a), number average molecular weight (b), weight average molecular weight (c) and polydispersity (d) as function of time for the second step of bimodal synthesis.

Symbols: experimental values; lines: model predictions

Despite the different reaction mode, the model predictions agree remarkably well with the experimental data. Notably, the model properly account for the huge heterogeneity of the polymer properties which result from the bimodal synthesis, corresponding to very high polydispersity values. The removal of hydrogen corresponds to a drastic increase of polymerization rate as well as sudden reduction of termination by chain transfer, resulting in a "bimodal" distribution of polymer properties in the particle. This reaction mode could be effectively used to adjust the overall polymer properties by tuning the operating conditions of each step; the design of such conditions can be easily carried out taking advantage of the model here developed given its prediction ability. 


\section{Conclusions}

In this work, a multigrain model was employed in order to characterize the polyethylene synthesis in slurry phase performed in a semibatch reactor at the single particle scale. The model includes all the relevant phenomena which contribute the final polymer properties: chemical kinetics (through a simple but exhaustive kinetic scheme), inter- and intraparticle mass transport limitations and equilibrium phase partitioning. Such single-particle model was also coupled with a proper reactor description by means of mass conservation equations, thus developing a reactor modeling tool.

Due to the mechanistic nature of the developed model, all involved parameters have a physical meaning and can be independently estimated. All the model parameters could be evaluated through correlations or from literature data, with the exception of the rate constants, whose values were obtained by direct fitting of experimental data. This way, not only a satisfactorily quantitative description of the system was achieved, but also the effect of hydrogen on catalyst activity and polymer molecular weight was elucidated.

The model was finally validated against independent data sets: the good agreement between experiments and simulations for different process conditions confirmed the general reliability of the modeling approach and the proposed procedure for estimating kinetic parameters. Moreover, a simple two-site model for the catalyst proved to provide a satisfactory description of the system, in terms of average polymer properties.

\section{Acknowledgments}

GS acknowledges financial support from the Swiss National Science Foundation (SNSF), with Grant Number 200021_153403.

The authors acknowledge the contribution of Nic. Friederichs for paper revision.

\section{List of symbols}

[cocat]

$\left[\mathrm{H}_{2}\right]$

[M]

$\left[M_{i}\right]_{\mu p}$

$\left[M_{i}\right]_{b}$

$\left[M_{i}\right]_{b, e q}$

$\left[M_{i}\right]_{m p}$

$\left[M_{i}\right]_{m p, j}$

$\left[M_{i}\right]_{m p, i n t}$

[Me]

$C_{d}^{k}$

$C_{p H 2}$

$C_{p}{ }^{0}$
Cocatalyst concentration, $\mathrm{mol} \mathrm{cm}^{-3}$

Hydrogen concentration on crystal surface, $\mathrm{mol} \mathrm{cm}_{\mu \mathrm{p}}{ }^{-3}$

Monomer concentration on crystal surface, $\mathrm{mol} \mathrm{cm}_{\mu \mathrm{p}}{ }^{-3}$

Concentration of the i-th specie in microparticle, $\mathrm{mol} \mathrm{cm}_{\mu \mathrm{p}}{ }^{-3}$

Concentration of the i-th specie in liquid phase, $\mathrm{mol} \mathrm{cm}^{-3}$

Concentration of the i-th specie in liquid phase at equilibrium, $\mathrm{mol} \mathrm{cm}^{-3}$

Concentration of the i-th specie in macroparticle, $\mathrm{mol} \mathrm{cm}_{\mathrm{mp}}{ }^{-3}$

Concentration of the i-th specie in macroparticle j-th grid point, $\mathrm{mol} \mathrm{cm}_{\mathrm{mp}}{ }^{-3}$

Concentration of the i-th specie at macroparticle/liquid interface, $\mathrm{mol} \mathrm{cm}_{\mathrm{mp}}{ }^{-3}$

Metal concentration, $\mathrm{mol} \mathrm{cm}_{\mu \mathrm{p}}{ }^{-3}$

Fraction of spontaneously deactivated sites of type k, mol molMe $\mathrm{M}^{-1}$

Fraction of active sites inhibited by hydrogen, mol molMe${ }^{-1}$

Fraction of active metal, mol molMe ${ }^{-1}$ 


\begin{tabular}{|c|c|}
\hline$C_{p}{ }^{k}$ & Fraction of potentially active sites of type k not yet activated, mol molMe ${ }^{-1}$ \\
\hline$d_{c a t}$ & Initial catalyst size, $\mathrm{cm}$ \\
\hline$D_{i, \mu p}$ & Diffusion coefficient of the i-th specie in microparticle, $\mathrm{cm}^{2} \mathrm{~s}^{-1}$ \\
\hline$D_{i, b}$ & Diffusion coefficient of the i-th specie in liquid phase, $\mathrm{cm}^{2} \mathrm{~s}^{-1}$ \\
\hline$D_{i, m p}$ & Diffusion coefficient of the i-th specie in macroparticle, $\mathrm{cm}^{2} \mathrm{~s}^{-1}$ \\
\hline$D_{n}{ }^{k}$ & Dead chain of length n produced on active site of type $\mathrm{k}$, mol molMe $\mathrm{e}^{-1}$ \\
\hline$D P_{\text {inst }}^{k}$ & Instantaneous degree of polymerization of polymer produced at active site k, - \\
\hline$F$ & Objective function, - \\
\hline$H_{i}$ & Henry coefficient of the i-th species, Pa \\
\hline$k_{\text {act }}{ }^{k}$ & Activation kinetic constant for active site of type $\mathrm{k}, \mathrm{s}^{-1}$ \\
\hline$k_{c, i}$ & Solid/liquid mass transfer coefficient of the i-th species, $\mathrm{cm} \mathrm{s}^{-1}$ \\
\hline$k_{\text {deact }}{ }^{k}$ & Spontaneous deactivation kinetic constant for active site of type $\mathrm{k}, \mathrm{s}^{-1}$ \\
\hline$k_{l, i}$ & Gas/liquid mass transfer coefficient of the i-th species, $\mathrm{cm} \mathrm{s}^{-1}$ \\
\hline$k_{p}{ }^{k}$ & Propagation kinetic constant for active site of type $\mathrm{k}, \mathrm{cm}_{\mu \mathrm{p}}{ }^{3} \mathrm{~mol}^{-1} \mathrm{~s}^{-1}$ \\
\hline$k_{t H}{ }^{k}$ & Hydrogen chain transfer kinetic constant for active site of type $\mathrm{k}, \mathrm{cm}_{\mu \mathrm{p}}{ }^{3} \mathrm{~mol}^{-1} \mathrm{~s}^{-1}$ \\
\hline$k_{t M}{ }^{k}$ & Monomer chain transfer constant for active site of type $\mathrm{k}, \mathrm{cm}_{\mu \mathrm{p}}{ }^{3} \mathrm{~mol}^{-1} \mathrm{~s}^{-1}$ \\
\hline$M_{i, j}$ & Concentration of the i-th species in the $\mathrm{j}$-th grid point, $\mathrm{mol} \mathrm{cm}_{\mathrm{mp}}{ }^{-3}$ \\
\hline$M W_{M e}$ & Molecular weight of the active metal, $\mathrm{g} \mathrm{mol}^{-1}$ \\
\hline$M W_{\text {mon }}$ & Monomer molecular weight, $\mathrm{g} \mathrm{mol}^{-1}$ \\
\hline$M W_{n}$ & Number average molecular weight, $\mathrm{g} \mathrm{mol}^{-1}$ \\
\hline$M W_{n, a v e}$ & Volume averaged number average molecular weight, $\mathrm{g} \mathrm{mol}^{-1}$ \\
\hline$M W_{n, \text { norm }}$ & Normalized volume averaged number average molecular weight, - \\
\hline$M W_{w}$ & Weight average molecular weight, g mol${ }^{-1}$ \\
\hline$M W_{w, a v e}$ & Volume averaged weight average molecular weight, $\mathrm{g} \mathrm{mol}^{-1}$ \\
\hline$M W_{w, n o r m}$ & Normalized volume averaged weight average molecular weight, - \\
\hline$n_{\text {exp }}$ & Number of experimental points \\
\hline$N_{i}$ & Number of moles of the i-th species in gas phase, mol \\
\hline$N_{i, j}$ & Number of moles of the i-th species in the j-th grid point, mol \\
\hline$N_{\text {part }}$ & Number of particles in liquid phase, \# \\
\hline$P_{i}$ & Partial pressure of the i-th species in gas phase, $\mathrm{Pa}$ \\
\hline$P_{i}^{0}$ & Solvent vapor pressure, $\mathrm{Pa}$ \\
\hline$P_{0}{ }^{k}$ & Vacant site of type $\mathrm{k}$, mol molMe $\mathrm{e}^{-1}$ \\
\hline$P_{n}{ }^{k}$ & Living chain with $\mathrm{n}$ monomeric units growing in an active site of type $\mathrm{k}$, mol molMe${ }^{-1}$ \\
\hline$P_{\text {tot }}{ }^{k}$ & Sum of vacant and occupied active sites of type $\mathrm{k}$, mol molMe ${ }^{-1}$ \\
\hline$P D$ & Polydispersity, - \\
\hline$P D_{\text {ave }}$ & Volume averaged polydispersity, - \\
\hline$P D_{\text {inst }}{ }^{k}$ & Instantaneous volume averaged polydispersity for polymer produced at active site k, - \\
\hline
\end{tabular}




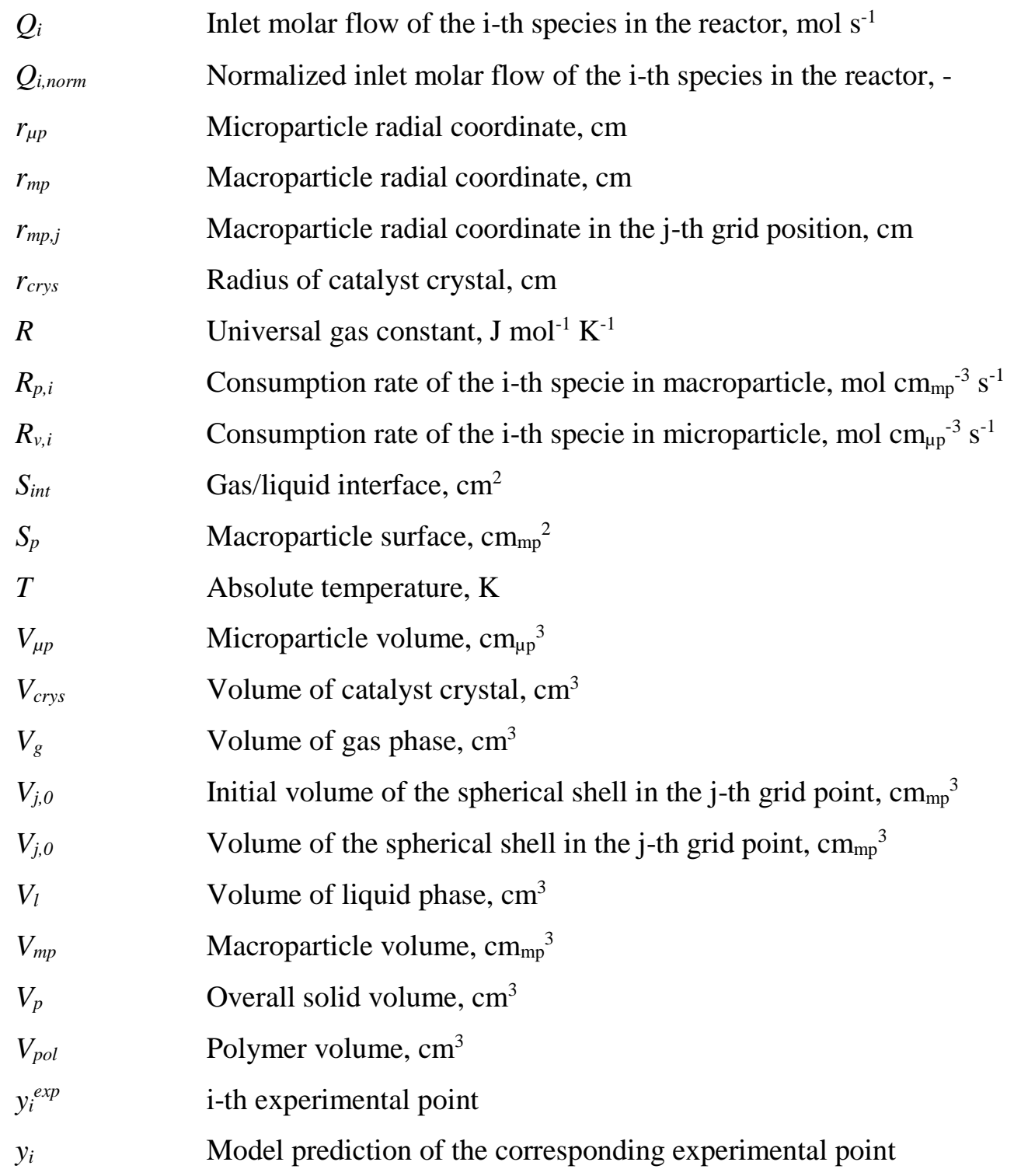

\section{Greek symbols}

$\alpha$

$\varepsilon$

$\lambda_{l}^{k}$

$\mu_{l}^{k}$

$\mu_{l, a v e}$

$\eta_{i}$

$\phi$

$\phi_{j}$

$\rho_{\text {cat }}$

$\tau$

$\omega_{M e}$
Fraction of active sites of type 1, -

Macroparticle void fraction, -

l-th order moment of growing chains on active site of type $\mathrm{k}$, mol molMe-1

l-th order moment of bulk chains produced in active site of type $\mathrm{k}$, mol molMe${ }^{-1}$

volume averages l-th order moment of bulk chains, mol molMe ${ }^{-1}$

Partition factor of the i-th species, -

Growth factor, -

Growth factor in the j-th grid point, -

Catalyst density, $\mathrm{g} \mathrm{cm}^{-3}$

Catalyst tortuosity, -

Mass fraction of active metal, - 


$\begin{array}{ll}\text { Subscripts } & \\ \mu p & \text { Microparticle } \\ \text { i } & \text { i-th species } \\ \text { j } & \text { j-th grid point } \\ \text { l } & \text { l-th order moment } \\ \text { mp } & \text { Macroparticle }\end{array}$

[1] L. L. Bohm, Angew Chem Int Edit 2003, 42, 5010.

[2] V. Touloupidis, Macromol React Eng 2014, 8, 508.

[3] D. Singh, R. P. Merrill, Macromolecules 1971, 4, 599.

[4] V. Kanellopoulos, G. Dompazis, B. Gustafsson, C. Kiparissides, Ind Eng Chem Res 2004, 43, 5166.

[5] T. F. Mckenna, J. Dupuy, R. Spitz, J Appl Polym Sci 1995, 57, 371.

[6] M. A. Dube, J. B. P. Soares, A. Penlidis, A. E. Hamielec, Ind Eng Chem Res 1997, 36, 966.

[7] T. F. McKenna, J. B. P. Soares, Chem Eng Sci 2001, 56, 3931.

[8] R. Galvan, M. Tirrell, Chem Eng Sci 1986, 41, 2385.

[9] S. Floyd, R. A. Hutchinson, W. H. Ray, J Appl Polym Sci 1986, 32, 5451.

[10] R. A. Hutchinson, C. M. Chen, W. H. Ray, J Appl Polym Sci 1992, 44, 1389.

[11] D. Ramkrishna, "Population balances : theory and applications to particulate systems in engineering", Academic Press, San Diego, CA, 2000, p. xvi.

[12] H. Hatzantonis, A. Goulas, C. Kiparissides, Chem Eng Sci 1998, 53, 3251.

[13] J. B. P. Soares, A. E. Hamielec, Macromol Theor Simul 1995, 4, 1085.

[14] J. J. Zacca, J. A. Debling, W. H. Ray, Chem Eng Sci 1997, 52, 1941.

[15] A. Alizadeh, M. Namkajorn, E. Somsook, T. F. L. McKenna, Macromol Chem Phys 2015, 216, 903.

[16] A. Alizadeh, M. Namkajorn, E. Somsook, T. F. L. McKenna, Macromol Chem Phys 2015, 216, 985.

[17] C. H. Fontes, M. J. Mendes, Polymer 2005, 46, 2922.

[18] M. S. Bhagwat, S. S. Bhagwat, M. M. Sharma, Ind Eng Chem Res 1994, 33, 2322.

[19] V. Touloupides, V. Kanellopoulos, P. Pladis, C. Kiparissides, D. Mignon, P. Van-Grambezen, Chem Eng Sci 2010, 65, 3208.

[20] A. G. M. Neto, M. F. Freitas, M. Nele, J. C. Pinto, Ind Eng Chem Res 2005, 44, 2697.

[21] J. Z. Zhao, J. Z. Sun, Q. Y. Zhou, Z. R. Pan, J Appl Polym Sci 2001, 81, 719.

[22] G. Dompazis, V. Kanellopoulos, C. Kiparissides, Macromol Mater Eng 2005, 290, 525.

[23] J. B. P. Soares, A. E. Hamielec, Polym React Eng 1996, 4, 153.

[24] J. T. McCoy, R. Rawatlal, Comput Chem Eng 2012, 36, 68.

[25] M. J. H. Khan, M. A. Hussain, Z. Mansourpour, N. Mostoufi, N. M. Ghasem, E. C. Abdullah, J Ind Eng Chem 2014, 20, 3919.

[26] Y. Che, Z. Tian, Z. Liu, R. Zhang, Y. X. Gao, E. G. Zou, S. H. Wang, B. P. Liu, Powder Techno/ 2015, 286, 107.

[27] J. B. P. Soares, A. E. Hamielec, Polymer 1995, 36, 2257.

[28] J. A. Debling, W. H. Ray, Ind Eng Chem Res 1995, 34, 3466.

[29] M. Ahmadi, M. Nekoomanesh, H. Arabi, Macromol React Eng 2010, 4, 135.

[30] Y. V. Kissin, F. M. Mirabella, C. C. Meverden, J Polym Sci Pol Chem 2005, 43, 4351.

[31] K. R. Chen, Z. Tian, N. Luo, B. P. Liu, Ind Eng Chem Res 2014, 53, 19905.

[32] J. B. P. Soares, Chem Eng Sci 2001, 56, 4131.

[33] K. Z. Yao, B. M. Shaw, B. Kou, K. B. McAuley, D. W. Bacon, Polym React Eng 2003, 11, 563.

[34] J. B. P. Soares, T. F. Mckenna, "Polyolefin Reaction Engineering", Wiley, 2012.

[35] F. Gemoets, M. Zhang, T. W. Karjala, B. W. S. Kolthammer, Macromol React Eng 2010, 4, 109.

[36] T. Garoff, S. Johansson, K. Pesonen, P. Waldvogel, D. Lindgren, Eur Polym J 2002, 38, 121.

[37] Y. V. Kissin, R. I. Mink, T. E. Nowlin, J Polym Sci Pol Chem 1999, 37, 4255.

[38] Y. V. Kissin, J Polym Sci Pol Chem 2001, 39, 1681. 
[39] Y. V. Kissin, Macromol Theor Simul 2002, 11, 67.

[40] P. Varshney, D. Kunzru, S. K. Gupta, Chem Eng Res Des 2010, 88, 455.

[41] K. S. Ha, K. Y. Yoo, H. K. Rhee, J Appl Polym Sci 2001, 79, 2480.

[42] Y. Chen, X. G. Liu, Polymer 2005, 46, 9434.

[43] R. J. LeVeque, "Finite difference methods for ordinary and partial differential equations : steady-state and time-dependent problems", Society for Industrial and Applied Mathematics, Philadelphia, PA, 2007, p. $\mathrm{xv}$.

[44] S. Floyd, K. Y. Choi, T. W. Taylor, W. H. Ray, J Appl Polym Sci 1986, 31, 2231. 